\title{
The Chapman-Jouguet closure for the Riemann problem with vaporization
}

\author{
Vincent Perrier $^{\dagger}$ \\ January 10, 2008
}

\begin{abstract}
This work is devoted to the modelling of phase transition. The thermodynamic model for phase transition chosen is a model with two equations of state, each of them modelling one phase of a given fluid. The mixture equation of state is obtained by an entropy optimization criterion. Both equations of state are supposed to be convex and a necessary condition is found to ensure the convexity of the mixture equation of state. Then we investigate the Riemann problem for the Euler system with these equations of state. More precisely, we propose to take into account metastable states, which may occur as remarked in [13]. We check that the ChapmanJouguet theory can be applied in our context, and that it is consistent with the entropy growth criterion. As the characteristic Lax criterion does not hold for this solution, an additional relation, the kinetic closure is necessary. The common closure, i.e. the Chapman-Jouguet closure is proved to be uncorrect in general in that context.
\end{abstract}

\section{Introduction}

We are interested in the study of some problems arising in the modelling of phase transition in compressible fluids. A widely used model for phase transition $[2,1,14,5]$ is the Van-Der-Waals'one. Nevertheless the very physical meaning of this model is questionable, because the resulting system of partial derivative equations is not hyperbolic. Moreover according to [16], the shock structure found in [14] does not seem to match with what experiments show. Another approach consists in modelling each phase by an equation of state, and in coupling them by optimizing the entropy $[7,9]$ to get a mixture equation of state. As explained in [12], the convexity of internal energy is necessary to ensure the local thermodynamic equilibrium. Whether the mixture equation of state is convex or not will be discussed in a first part.

\footnotetext{
†perrier@math.u-bordeaux1.fr, Université Bordeaux 1, Mathématiques Appliquées de Bordeaux and CEntre des Lasers Intenses et Applications, 33405 Talence Cedex
} 


\begin{tabular}{|c|l|}
\hline$T$ & temperature \\
$\tau$ & specific volume \\
$\rho$ & density \\
$s$ & specific entropy \\
$\mu$ & chemical potential \\
$h$ & specific enthalpy \\
\hline
\end{tabular}

\begin{tabular}{|c|l|}
\hline$f$ & specific free energy \\
$P$ & pressure \\
$\varepsilon$ & specific internal energy \\
$y$ & mass fraction \\
$\alpha$ & volume fraction \\
\hline
\end{tabular}

Table 1: Thermodynamic notations

We will then concentrate on the Euler system of partial derivative equations, which models flow dynamics without viscosity nor thermal conduction. A fundamental step to approximate the solutions of the Euler system with a Godunov' method is to solve the Riemann problem, i.e. the Cauchy problem where the initial condition is composed of two differents constant states. If the solution of the Riemann problem for the Euler system is easy to solve for convex equation of state, it becomes much harder when the equation of state suffers from loss of derivative and from local non-convexity $[19,12,20,21,16,17,18,11]$, because the common entropy growth criterion fails to ensure the existence and uniqueness of the solution. Based on the experiments of [13], we propose to take into account out of thermodynamic equilibrium states, or metastable states. The Chapman-Jouguet theory will be used $[4,6]$, and the compatibility between the model of equation of state and that theory will be discussed. The entropy growth criterion will be shown to hold. Finally we will show that the closure usually used to close the problem [10] i.e. the Chapman-Jouguet closure leads to a solution that does not depend continuously in general on its initial data, and this solution will therefore be rejected.

\section{Thermodynamic preliminaries}

\subsection{Thermodynamic with phase transition}

We suppose that we have two phases of the same fluid. For the sake of simplicity, we will consider that one phase is liquid (subscript $l$ ) and the other one is the vapor (subscript $v$ ). For each phase, we will take the notations of Table 1 . If we consider that we have a mixture of the two phases, then the total specific quantities are defined by

$$
\begin{aligned}
& \tau_{t o t}=y_{l} \tau_{l}+y_{v} \tau_{v}, \\
& \varepsilon_{t o t}=y_{l} \varepsilon_{l}+y_{v} \varepsilon_{v}, \\
& s_{t o t}=y_{l} s_{l}+y_{v} s_{v} .
\end{aligned}
$$

To find the thermodynamic equilibrium, the total entropy (1c) must be optimized. Of course, the optimization must be consistent with the following 
constraints:

$$
\begin{array}{cl}
\text { conservation of total energy } & y_{l} \varepsilon_{l}+y_{v} \varepsilon_{v}=\text { cste, } \\
\text { conservation of mass } & y_{l}+y_{v}=1 .
\end{array}
$$

Moreover, we suppose that the two phases are locally non miscible, which means that

$$
y_{l} \tau_{l}+y_{v} \tau_{v}=\text { cste. }
$$

We choose to optimize (1c) with the variables $\tau, \varepsilon, y$ for each phase. The first and second principle of thermodynamic impose that for each phase

$$
\mathrm{d} s=\frac{\mathrm{d} \varepsilon}{T}+\frac{P}{T} \mathrm{~d} \tau .
$$

Then the differential of $s_{t o t}$ must belong to the set spanned by the gradients of the constraints; if we denote by $\lambda_{1}, \lambda_{2}, \lambda_{3}$ the Lagrange multipliers associated to the constraints $(2 \mathrm{a}),(2 \mathrm{~b}),(2 \mathrm{c})$, we find (with the notations of 1 )

$$
\begin{aligned}
s_{l} & =\lambda_{1} \varepsilon_{l}+\lambda_{2}+\lambda_{3} \tau_{l}, \\
\frac{y_{l}}{T_{l}} & =\lambda_{1} y_{l}, \\
\frac{y_{l} P_{l}}{T_{l}} & =\lambda_{3} y_{l}, \\
s_{v} & =\lambda_{1} \varepsilon_{v}+\lambda_{2}+\lambda_{3} \tau_{v}, \\
\frac{y_{v}}{T_{v}} & =\lambda_{1} y_{v}, \\
\frac{y_{v} P_{v}}{T_{v}} & =\lambda_{3} y_{v} .
\end{aligned}
$$

If we suppose that both phases coexist, then equations (3b) and (3e) give

$$
T_{l}=T_{v}=: T,
$$

and equations (3c) and (3f) give

$$
P_{l}=P_{v}=: P,
$$

and finally, (3a) and (3d) lead to

$$
\mu_{l}(P, T)=\mu_{v}(P, T) .
$$

It is well known that there exist a temperature $T_{c}$ and a pressure $P_{c}$ such that there is no more difference between the liquid and the gas above these temperature and pressure (the fluid is said to be supercritical). If we suppose that under these values we have

$$
\tau_{l}(P, T) \neq \tau_{v}(P, T),
$$




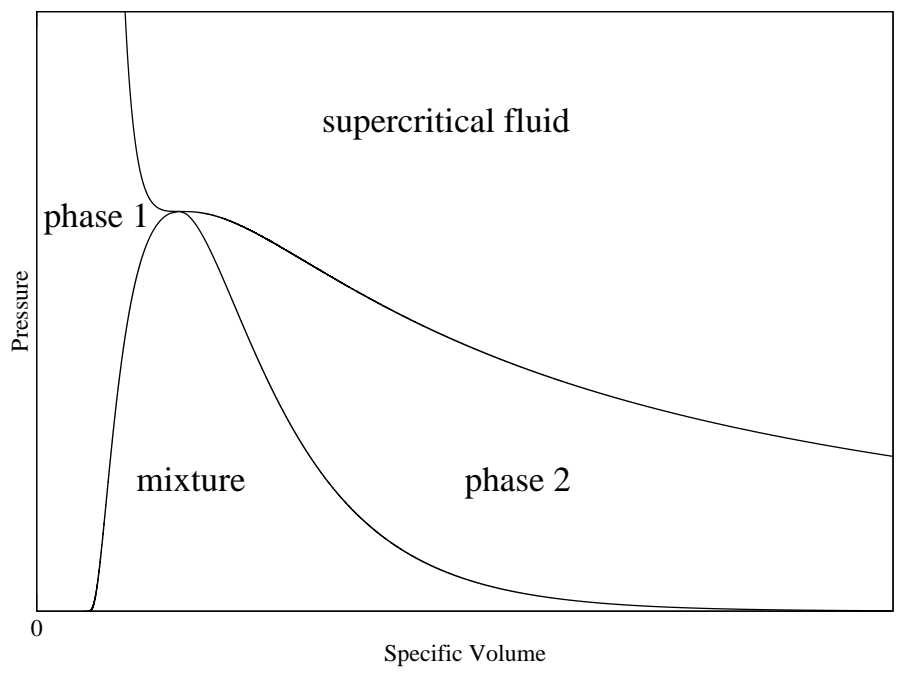

Figure 1: The thermodynamic plane $(P, \tau)$ is divided into four parts : two parts where the single phases are stable, one part where the mixture is stable, and one part where the fluid is supercritical

then we can apply the implicit functions theorem to (4) to prove that $P$ is locally a function of $T$

$$
P=P_{\text {sat }}(T) .
$$

The limit of thermodynamic stability of the mixture is given, in the $(\tau, P)$ plane by the functions $\tau_{v}\left(P_{\text {sat }}(T), T\right)$ and $\tau_{l}\left(P_{\text {sat }}(T), T\right)$. The set of all the physical states lying between these curves is called the saturation dome. When there is no ambiguity, we will also denote by $\tau_{v}$ the function $T \mapsto \tau_{v}\left(P_{s a t}(T), T\right)$ and by $\tau_{l}$ the function $T \mapsto \tau_{l}\left(P_{s a t}(T), T\right)$. Thus, the thermodynamic plane can be divided into four parts (see Figure 1) : two parts in which one of the two pure phases is stable, one part where a mixture of saturated liquid and vapor is stable, and where the specific thermodynamic variables are defined as in (1), and one part with the supercritical fluid.

Moreover, if we differentiate the equality $\mu_{v}(P, T)=\mu_{l}(P, T)$ with respect to $(P, T)$, we find the Clausius-Clapeyron relation

$$
\frac{\mathrm{d} P_{\text {sat }}}{\mathrm{d} T}(T)=\frac{s_{v}(T)-s_{l}(T)}{\tau_{v}(T)-\tau_{l}(T)} .
$$

For most of the solid-liquid phase transition (except for special cases like bismuth or water) and for all the liquid-gas phase transition, we have, for all $T<T_{c}$

$$
\tau_{v}(T)-\tau_{l}(T)>0
$$

This means that in the Figure 1, the phase 1 is the liquid, and the phase 2 is a gas. 
For any phase transition, the entropy of the most compact constituent is lower than the entropy of the other constituent. Thus in our case we have for all $T<T_{c}$

$$
s_{v}(T)-s_{l}(T)>0
$$

which induces $\frac{\mathrm{d} P_{\text {sat }}}{\mathrm{d} T}>0$.

\subsection{Adimensioned thermodynamic coefficients}

We adopted the notations of the Table 1. As in [12], we define three adimensioned parameters as

$$
\Gamma=-\frac{\tau}{T}\left(\frac{\partial T}{\partial \tau}\right)_{s}, \quad \gamma=-\frac{\tau}{P}\left(\frac{\partial P}{\partial \tau}\right)_{s}, \quad g=\frac{P \tau}{T^{2}}\left(\frac{\partial T}{\partial s}\right)_{\tau} .
$$

$\gamma$ is called the adiabatic coefficient, and $\Gamma$ is the Grüneisen coefficient. As done in [12], we propose to use these three thermodynamic coefficients to express all the thermodynamic quantities. One can then show that the following identities hold

$$
\begin{aligned}
\mathrm{d} s & =\frac{P \tau}{T^{2}} \frac{1}{g} \mathrm{~d} T+\frac{P}{T} \frac{\Gamma}{g} \mathrm{~d} \tau, \\
\mathrm{d} s & =-\frac{\tau}{T} \frac{\Gamma}{\gamma g-\Gamma^{2}} \mathrm{~d} P+\frac{P \tau}{T^{2}} \frac{\gamma}{\gamma g-\Gamma^{2}} \mathrm{~d} T, \\
\mathrm{~d} s & =\frac{\tau}{T} \frac{1}{\Gamma} \mathrm{d} P+\frac{P}{T} \frac{\gamma}{\Gamma} \mathrm{d} \tau, \\
\mathrm{d} \tau & =-\frac{\tau}{P} \frac{g}{\gamma g-\Gamma^{2}} \mathrm{~d} P+\frac{\tau}{T} \frac{\Gamma}{\gamma g-\Gamma^{2}} \mathrm{~d} T, \\
\mathrm{~d} h & =\tau \frac{\Gamma+1}{\Gamma} \mathrm{d} P+P \frac{\gamma}{\Gamma} \mathrm{d} \tau, \\
\mathrm{d} \varepsilon & =\tau \frac{1}{\Gamma} \mathrm{d} P+P \frac{(\gamma-\Gamma)}{\Gamma} \mathrm{d} \tau .
\end{aligned}
$$

Thermodynamic stability requires that $\varepsilon$ be convex as a function of $\tau$ and $s$, which leads to

$$
g \geq 0, \quad \gamma \geq 0, \quad \gamma g-\Gamma^{2} \geq 0
$$

\subsection{The fundamental derivative}

In [15], the fundamental derivative was defined as

$$
\mathscr{G}=-\frac{\tau}{2} \frac{\left(\frac{\partial^{3} \varepsilon}{\partial \tau^{3}}\right)_{s}}{\left(\frac{\partial^{2} \varepsilon}{\partial \tau^{2}}\right)_{s}}
$$

The sign of $\mathscr{G}$ determines whether the Hugoniot curve and the isentropes are convex or not in the $(\tau, P)$ plane. We will suppose in the following that $\mathscr{G}$ is 
positive, so that no undercompressive discontinuity nor expansion fans can exist (see $[15,20,21])$.

\subsection{Mixture equation of state}

\subsubsection{Parameterization of the mixture equation of state}

In the following, we will denote with a subscript $m$ all the variables relative to the mixture equation of state. The mixture equation of state is naturally parameterized by $y$, the mass fraction of the gas, and $T$, the temperature. Nevertheless, in the next sections, the parameters that will intervene are mostly $\tau$ and $s$. They are linked by the transformation

$$
\Phi:\left(\begin{array}{c}
y \\
T
\end{array}\right) \mapsto\left(\begin{array}{c}
y \tau_{v}(T)+(1-y) \tau_{l}(T) \\
y s_{v}(T)+(1-y) s_{l}(T)
\end{array}\right)=\left(\begin{array}{c}
\tau \\
s
\end{array}\right) .
$$

Theorem 1. For all points in the saturation dome, $\Phi$ is a local diffeomorphism provided the equations of state of the liquid and of the gas are both convex.

Proof. To show that $\Phi$ is a local diffeomorphism, it is sufficient to show that its Jacobian does not vanish. Differentiation of (9) and using the ClausiusClapeyron relation leads to

$$
\operatorname{det}(\mathrm{D} \Phi)=\left(\tau_{v}-\tau_{l}\right)\left(y\left(\frac{\mathrm{d} s_{v}}{\mathrm{~d} T}-\frac{\mathrm{d} P_{s a t}}{\mathrm{~d} T} \frac{\mathrm{d} \tau_{v}}{\mathrm{~d} T}\right)+(1-y)\left(\frac{\mathrm{d} s_{l}}{\mathrm{~d} T}-\frac{\mathrm{d} P_{s a t}}{\mathrm{~d} T} \frac{\mathrm{d} \tau_{l}}{\mathrm{~d} T}\right)\right) .
$$

We supposed that $\tau_{v}-\tau_{l}>0$ (except at the critical point), so that there remains to show that

$$
y\left(\frac{\mathrm{d} s_{v}}{\mathrm{~d} T}-\frac{\mathrm{d} P_{s a t}}{\mathrm{~d} T} \frac{\mathrm{d} \tau_{v}}{\mathrm{~d} T}\right)+(1-y)\left(\frac{\mathrm{d} s_{l}}{\mathrm{~d} T}-\frac{\mathrm{d} P_{s a t}}{\mathrm{~d} T} \frac{\mathrm{d} \tau_{l}}{\mathrm{~d} T}\right),
$$

never vanishes. The term (11) is a convex combination of

$$
\frac{\mathrm{d} s_{v}}{\mathrm{~d} T}-\frac{\mathrm{d} P_{s a t}}{\mathrm{~d} T} \frac{\mathrm{d} \tau_{v}}{\mathrm{~d} T} \quad \text { and } \quad \frac{\mathrm{d} s_{l}}{\mathrm{~d} T}-\frac{\mathrm{d} P_{s a t}}{\mathrm{~d} T} \frac{\mathrm{d} \tau_{l}}{\mathrm{~d} T} .
$$

Using the equations (3a),(3b) for $P=P_{\text {sat }}(T)$ leads to

$$
\frac{\mathrm{d} s_{b}}{\mathrm{~d} T}-\frac{\mathrm{d} P_{s a t}}{\mathrm{~d} T} \frac{\mathrm{d} \tau_{b}}{\mathrm{~d} T}=\frac{\gamma g-\Gamma^{2}}{g} \frac{P}{\tau}\left(\frac{\mathrm{d} \tau_{b}}{\mathrm{~d} T}\right)^{2}+\frac{P \tau}{T^{2}} \frac{1}{g}>0,
$$

for $b=g$ or $l$, which is positive provided each pure phase equation of state is convex.

Therefore (11) is positive, because it is a convex combination of two terms like (13). As a consequence, $\operatorname{det}(\mathrm{D} \Phi)>0$.

Thanks for the parameterization (9), we can calculate the adimensioned coefficients defined by (6), to show that 
Theorem 2. If both equation of state are convex, and if $\frac{\mathrm{d} P_{\text {sat }}}{\mathrm{d} T}>0$ then the mixture equation of state is convex too, i.e. inequalities (8) hold.

Proof. We denote by a subscript $m$ the thermodynamic parameters relative to the mixture equation of state.

- Calculation of $\Gamma_{m}$. To calculate $\Gamma_{m}$, we first use the chain rule

$$
\left(\frac{\partial \tau}{\partial T}\right)_{s}=\left(\frac{\partial y}{\partial T}\right)_{s}\left(\frac{\partial \tau}{\partial y}\right)_{T}+\left(\frac{\partial \tau}{\partial T}\right)_{y}
$$

Then the differentiation of the definition of mixture entropy shows that

$$
\left(\frac{\partial y}{\partial T}\right)_{s}=\frac{y \frac{\mathrm{d} s_{v}}{\mathrm{~d} T}+(1-y) \frac{\mathrm{d} s_{l}}{\mathrm{~d} T}}{s_{l}-s_{v}}
$$

which leads to

$$
\left(\frac{\partial \tau}{\partial T}\right)_{s}=-\frac{y \frac{\mathrm{d} s_{v}}{\mathrm{~d} T}+(1-y) \frac{\mathrm{d} s_{l}}{\mathrm{~d} T}}{s_{l}-s_{v}}\left(\tau_{l}-\tau_{v}\right)+y \frac{\mathrm{d} \tau_{v}}{\mathrm{~d} T}+(1-y) \frac{\mathrm{d} \tau_{l}}{\mathrm{~d} T} .
$$

Thanks for the Clausius-Clapeyron relation we find

$$
\left(\frac{\partial \tau}{\partial T}\right)_{s}=y\left(\frac{\mathrm{d} \tau_{v}}{\mathrm{~d} T}-\frac{\mathrm{d} T}{\mathrm{~d} P} \frac{\mathrm{d} s_{v}}{\mathrm{~d} T}\right)+(1-y)\left(\frac{\mathrm{d} \tau_{v}}{\mathrm{~d} T}-\frac{\mathrm{d} T}{\mathrm{~d} P} \frac{\mathrm{d} s_{l}}{\mathrm{~d} T}\right),
$$

which is negative according to what we did for the Jacobian of $\Phi$. Therefore

$$
\Gamma_{m}=-\frac{T}{\tau}\left(\frac{\partial \tau}{\partial T}\right)_{s} \geq 0
$$

- Calculation of $\gamma_{m}$. As $P=P_{\text {sat }}(T)$ in the saturation area, we have

$$
\left(\frac{\partial \tau}{\partial P}\right)_{s}=\frac{\mathrm{d} T_{s a t}}{\mathrm{~d} P}\left(\frac{\partial \tau}{\partial T}\right)_{s}
$$

Thus $\gamma_{m}=\Gamma_{m} \frac{T}{P}\left(\frac{\mathrm{d} P}{\mathrm{~d} T}\right)_{s a t} \geq 0$.

- Calculation of $g_{m}$. By using the identity

$$
\left(\frac{\partial T}{\partial s}\right)_{\tau}\left(\frac{\partial s}{\partial \tau}\right)_{T}\left(\frac{\partial \tau}{\partial T}\right)_{s}=-1
$$

we have

$$
\left(\frac{\partial T}{\partial s}\right)_{\tau}=-\frac{1}{\left(\frac{\partial s}{\partial \tau}\right)_{T}\left(\frac{\partial \tau}{\partial T}\right)_{s}}
$$


Along an isotherm we have $\mathrm{d} s=\left(s_{v}-s_{l}\right) \mathrm{d} y$, and $\mathrm{d} \tau=\left(\tau_{v}-\tau_{l}\right) \mathrm{d} y$, so that

$$
\left(\frac{\partial s}{\partial \tau}\right)_{T}=\left(\frac{\mathrm{d} P}{\mathrm{~d} T}\right)_{s a t}
$$

Therefore, we find

$$
\left(\frac{\partial T}{\partial s}\right)_{\tau}=\left(\frac{\mathrm{d} T}{\mathrm{~d} P}\right)_{s a t} \frac{\Gamma_{m} T}{\tau}
$$

which induces $\gamma_{m} g_{m}=\Gamma_{m}^{2}$. As $\gamma_{m} \geq 0$, this means that $g_{m} \geq 0$.

Therefore, we proved that $g_{m} \geq 0, \gamma \geq 0$, and that $\gamma_{m} g_{m}-\Gamma_{m}^{2}=0$, so that the convexity of energy is ensured.

\subsection{Adimensioned coefficients near a phase transition bound- ary}

In that section, we keep on denoting by the subscript $m$ the thermodynamic coefficients of the mixture equation of state, the coefficients with no subscript being the one of the pure phase.

In [12] (p.121), the following identity is proved

$$
\frac{\gamma-\gamma_{m}}{\gamma_{m}}=\left(\gamma g-\Gamma^{2}\right)\left(\frac{T}{\tau}\left(\frac{\mathrm{d} s_{b}}{\mathrm{~d} P}\right)_{s a t}\right)^{2}>0
$$

with $b=g$ or $l$. This identity proves that isentropes are stiffer in the pure phases than in the mixture. In the same manner it is proved that

$$
\frac{\Gamma_{m}}{\Gamma}=\frac{\gamma_{m}-\xi}{\gamma-\xi}
$$

with $\xi=-\frac{\tau}{P}\left(\frac{\mathrm{d} P}{\mathrm{~d} \tau}\right)_{\text {sat }}$. As in [12], we suppose that the isentropes can be parameterized by $\tau$, so that

$$
\frac{\gamma_{m}-\xi}{\gamma-\xi}>0
$$

so that $\Gamma$ is positive too, because $\Gamma_{m}>0$.

\subsection{Retrograde and Regular behavior}

In [16], the retrogradicity $r$ was introduced, to study the behavior of isentropes near a phase transition boundary

$$
r=\left(\frac{\partial T}{\partial \tau}\right)_{P}\left(\frac{\mathrm{d} s_{b}}{\mathrm{~d} P}\right)
$$

Thanks for $(7 \mathrm{~d})$ and as $\Gamma$ is positive near a phase transition boundary, $\left(\frac{\partial T}{\partial \tau}\right)_{P}$ is positive, so that the sign of $r$ is the same as the $\operatorname{sign}$ of $\frac{\mathrm{d} s_{b}}{\mathrm{~d} P}$. 

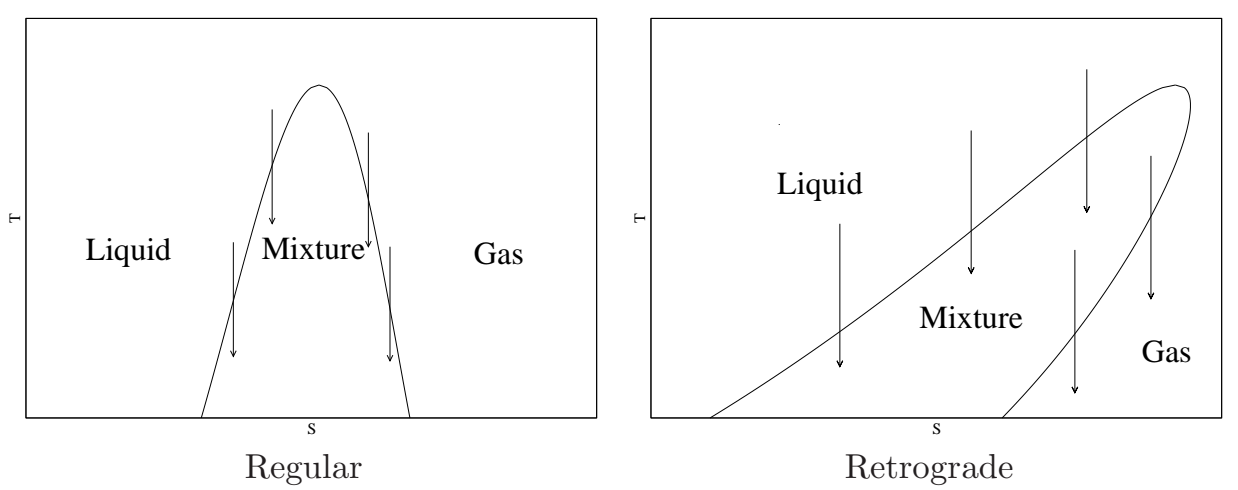

Figure 2: The saturation dome in the $(S, T)$ plane. On the left, the fluid is regular: all the isentropes (drawn as arrows) cross the saturation dome from the pure phase to the mixture. On the right, the fluid is retrograde: the isentropes are crossing the liquid saturation curve from the pure phase to the mixture, whereas it is the contrary on the gas side.

We suppose now that a fluid undergoes a rarefaction isentrope : this is the only regular transformation that a fluid can undergo. In the $(S, T)$ plane, this transformation is drawn as a vertical line. As the transformation is undercompressive, the temperature decreases (at least near the phase transition boundary, because $\Gamma>0$ and $\Gamma_{m}>0$ ). If $r>0$ then the isentrope crosses the saturation curve from the pure phase to to the mixture phase (as on both of the sides of the left Figure of Figure 2 and of the Liquid side of the right Figure of Figure 2). In that case, the fluid is said to be regular. If $r$ is negative, then the isentrope crosses the saturation curve from the mixture to the pure phase, as on the gas side of the right Figure of Figure 2.

In [12] (p.121), others expressions of $r$ are given

$$
r=\frac{\Gamma_{m}}{\Gamma} \frac{\gamma g-\Gamma^{2}}{\gamma_{m}} \frac{\mathrm{d} s_{b}}{\mathrm{~d} T}=\frac{\gamma-\gamma_{m}}{\gamma_{m}} \frac{\xi}{\xi-\gamma} .
$$

Experiments show that the liquid saturation curve is always regular. The gas saturation curve can be either regular or retrograde.

\subsection{Validity domain of a model}

To compute quickly a solution of the Riemann Problem for fluid flows, simplified equation of state (perfect gas of stiffened gas for example) are often preferred to tabulated ones. Nevertheless such equations of state have often only a narrow range of validity, out of which they do not have a physical behavior (negative energy, non convexity).

If we want to use a simplified EOS for both liquid and gas, we have to care not only about the physical behavior of the two EOS, but also about the mixture 
EOS computed. If we look at the properties needed in Section 1, we see that the property $\frac{\mathrm{d} P_{\text {sat }}}{\mathrm{d} T}>0$ is fundamental to ensure the local convexity of energy. Nevertheless, it is not always true as we show now on examples.

\subsubsection{Two perfect gas}

This model was proposed by $[8,7]$. The two phases are modeled with a perfect gas equation of state. To complete the equation of state, we suppose moreover that $C_{v}=1$ for each fluid. We denote by $\bar{\Gamma}_{i}$ the Grüneisen coefficient of the phase $i$. Then we have

$$
\begin{aligned}
& \varepsilon_{i}(P, \tau)=\frac{P \tau}{\bar{\Gamma}_{i}} \\
& s_{i}(P, T)=\log \left(T\left(\frac{\bar{\Gamma}_{i} T}{P}\right)^{\bar{\Gamma}_{i}}\right), \\
& \mu_{i}(P, T)=\left(\bar{\Gamma}_{i}+1\right) T-T \log \left(T\left(\frac{\bar{\Gamma}_{i} T}{P}\right)^{\bar{\Gamma}_{i}}\right) .
\end{aligned}
$$

The equation $\mu_{1}(P, T)=\mu_{2}(P, T)$ can be explicitly solved to get $P=\beta T$, with $\beta=\exp (1)\left(\frac{\Gamma_{2}^{\Gamma_{2}}}{\Gamma_{1}^{\Gamma_{1}}}\right)^{\frac{1}{\Gamma_{1}-\Gamma_{2}}}$. We see here that the condition $\frac{\mathrm{d} P_{\text {sat }}}{\mathrm{d} T}>0$ always holds. The limits of the saturation dome are given by the equations

$$
T=\varepsilon=\frac{P_{\text {sat }}(T) \tau_{i}(T)}{\Gamma_{i}}
$$

which gives $\tau_{i}(T)=\frac{\Gamma_{i}}{\beta}$. Thus, $T \mapsto \tau_{i}(T)$ is a constant function. In particular, the critical point does not exist. If we decide for example that $\Gamma_{1}<\Gamma_{2}$ then we get the projections of the phase diagram in the $(P, \tau)$ plane and in the $(S, T)$ plane that is drawn on Figure 3. The mixture equation of state can be explicitly calculated:

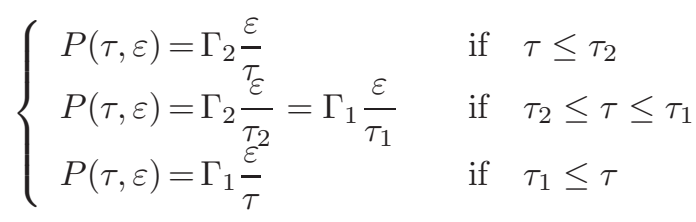

Nevertheless, we remark that the most heavy phase is described by the lowest adiabatic coefficient, which is in contradiction with what is described for example in [22] (chapter XI). Thus, the model with two perfect gas is a good mathematical model because the mixture equation of state can be explicitly calculated, but it cannot give a good account for the physic. 

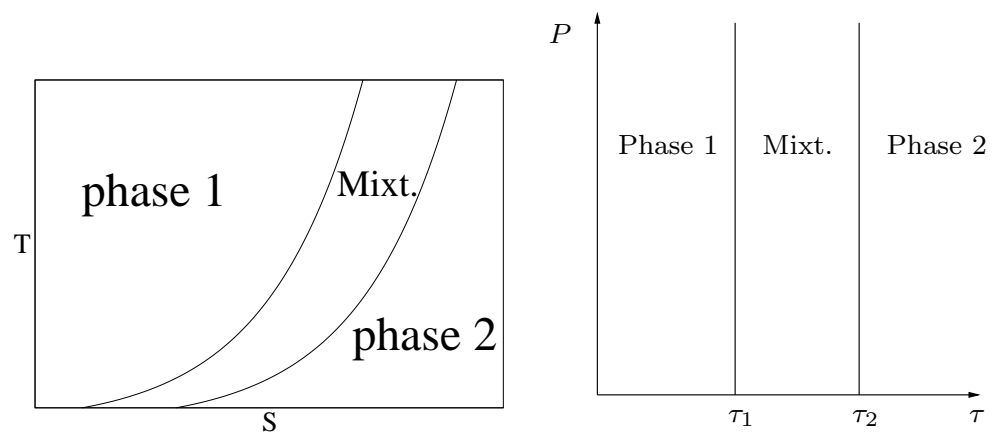

Figure 3: Shape of the saturation dome for two perfect gas. We note that the fluid is always retrograde.

\subsubsection{Two stiffened gas}

We model the two phases of a fluid with the Stiffened gas equation of state, for which we have (see [9])

$$
\begin{aligned}
& \varepsilon(P, \tau)=\frac{P+\bar{\gamma} P^{\infty}}{\bar{\gamma}-1} \tau+q \\
& s(P, T)=C_{v} \log \left(\frac{T^{\bar{\gamma}}}{\left(P+P^{\infty}\right)^{\bar{\gamma}-1}}\right)+q^{\prime} \\
& G(P, T)=\left(\bar{\gamma} C_{v}-q^{\prime}\right) T-C_{v} T \log \left(\frac{T^{\bar{\gamma}}}{\left(P+P^{\infty}\right)^{\bar{\gamma}-1}}\right)+q
\end{aligned}
$$

For this equation of state, the adimensioned coefficients are given by

$$
\gamma=\bar{\gamma}\left(1+\frac{P^{\infty}}{P}\right) \quad \Gamma=\bar{\gamma}-1 \quad g=\frac{(\bar{\gamma}-1) P}{P+P^{\infty}}
$$

$\gamma>0$ and $g>0$ are ensured if $\bar{\gamma}>1$. In [9], the coefficients $q, q^{\prime}, C_{v}, \bar{\gamma}, P^{\infty}$ were calculated for the gaseous and liquid phases to fit with the saturation curves near $T=298 \mathrm{~K}$. These coefficients are in Table 2 . The resulting function $P_{\text {sat }}(T)$ was drawn in Figure 4. For the two stiffened gas model, we cannot be sure that the functions $T \mapsto s_{v}(T)-s_{l}(T)$ and $T \mapsto \tau_{v}(T)-\tau_{l}(T)$ simultaneously vanish. Therefore, the critical point does not really exist. As we saw in the Section 1.4, we need that $\frac{\mathrm{d} P_{s a t}}{\mathrm{~d} T}>0$ to ensure the convexity of the mixture equation of state. Thus, the model is valid only when $\tau_{v}(T)-\tau_{l}(T)$ and $s_{v}(T)-s_{l}(T)$ are both positive. In our example, with the coefficients of Table 2 , the function $T \mapsto P_{\text {sat }}(T)$ are drawn on Figure 4 . We can see that the limit is near $T=970 \mathrm{~K}$, for which $\frac{\mathrm{d} P_{s a t}}{\mathrm{~d} T}$ vanishes. 


\begin{tabular}{|c|c|c|c|c|c|}
\hline Phase & $\bar{\gamma}$ & $P^{\infty}$ & $C_{v}$ & $q$ & $q^{\prime}$ \\
\hline Gas & 1.025 & 0 & 1956.45 & -237547. & -24485. \\
\hline Liquid & 2.35 & $4.10^{8} \mathrm{~Pa}$ & 1077.7 & -755269 & 0. \\
\hline
\end{tabular}

Table 2: Thermodynamic coefficients for the liquid and gaseous phase of dodecane.

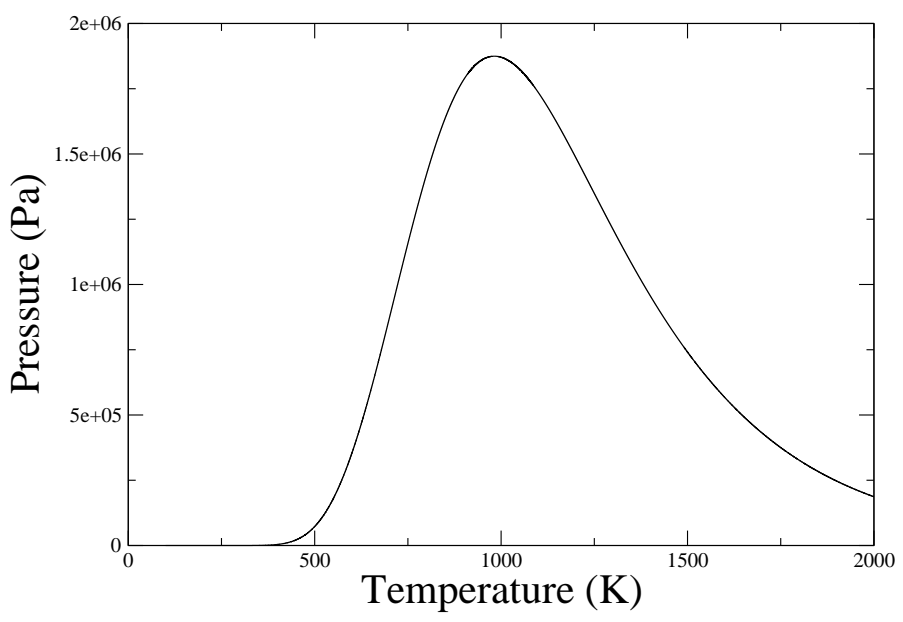

Figure 4: Numerical computation of the behavior of $P_{\text {sat }}(T)$ for two phases of stiffened-gas with the coefficients of Table 2. For temperatures below $970 \mathrm{~K}$, $T \mapsto P_{\text {sat }}(T)$ increases. For $T \approx 970 \mathrm{~K}$, the function $T \mapsto s_{v}(T)-s_{l}(T)$ vanishes and its sign changes, whereas the function $T \mapsto \tau_{v}(T)-\tau_{l}(T)$ does not vanish. As a consequence, $T \mapsto P_{\text {sat }}(T)$ does not increase any more and the equation of state is no more valid. 


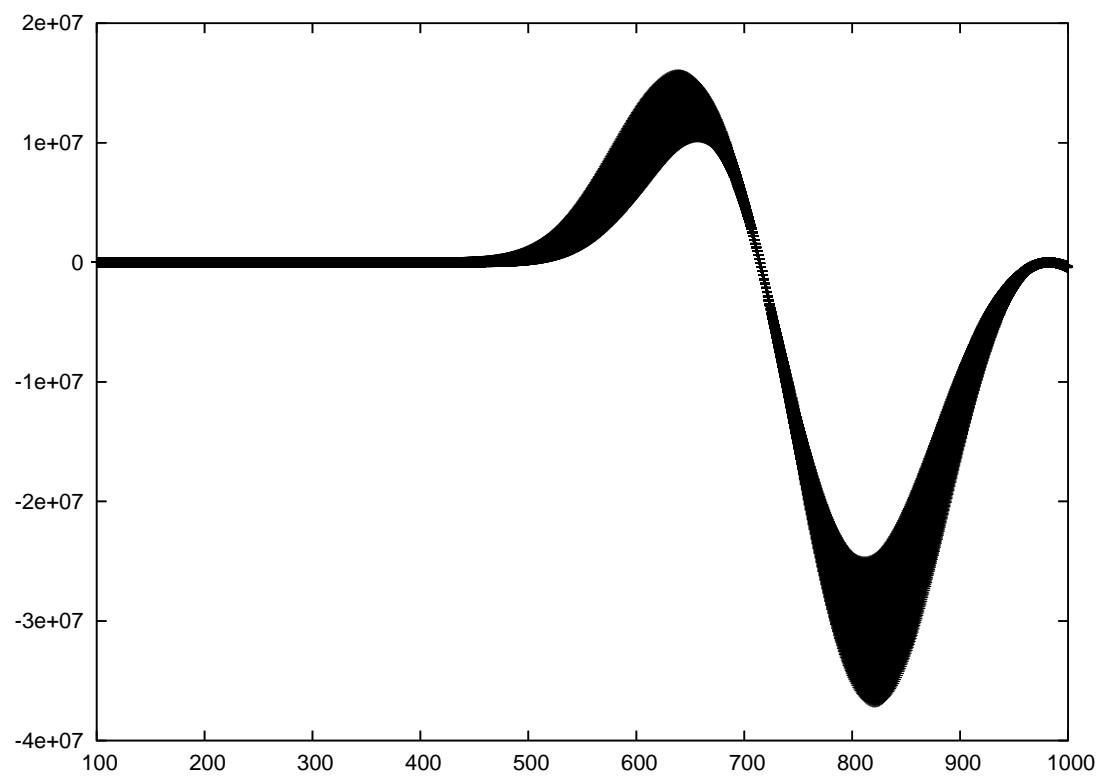

Figure 5: In this Figure, we draw all the $T \mapsto \mathscr{G}$ for $y \in[0 ; 1]$. The equation of state is the one of Table 2 . For low temperatures $(T \leq 715 \mathrm{~K})$, the fundamental derivative is positive whereas for $715 \leq T \leq 970 \mathrm{~K}$ the fundamental derivative is negative.

\subsection{Positivity of the fundamental derivative}

In Section 1.4, we found some criteria to ensure the convexity of the mixture equation of state. Nevertheless, we did not manage to find a simple criterion that can ensure too the positivity of the fundamental derivative of the mixture equation of state. Actually, as it is shown numerically in the Figure 5 for the thermodynamic coefficients of the Table 2, the fundamental derivative of the mixture equation of state can be either positive or negative, even if both equation of states have a positive fundamental derivative. In that example, the equation of state can be considered as valid if $T \leq 715 \mathrm{~K}$, where $\mathscr{G}>0$.

\section{Reminds on the Chapman-Jouguet theory (see [6] pp 142-160)}

Based on the experiments of $[13,16,17]$, we propose to take into account out of thermodynamic equilibrium states for solving the Riemann problem. By "out of equilibrium states" we mean metastable states, or overheated states, i.e. pure fluids that have a pressure $P$ and a specific volume $\tau$ that lie in the saturation dome. Existence of such states is due to some phenomena such as 
capillarity for example. In $[13,16,17]$ it was observed that phase transition waves were self-similar waves, so that Rankine-Hugoniot relations hold across them: $[F(U)-\sigma U]=0$, where $\sigma$ is the velocity of the discontinuity. These relations can be put in the following form (see [6] for example)

$$
\begin{aligned}
& \dot{M}=\frac{u_{1}-u_{0}}{\tau_{1}-\tau_{0}}, \\
& \dot{M}^{2}=-\frac{P_{1}-P_{0}}{\tau_{1}-\tau_{0}}, \\
& \varepsilon_{1}-\varepsilon_{0}+\frac{1}{2}\left(P_{1}+P_{0}\right)\left(\tau_{1}-\tau_{0}\right)=0,
\end{aligned}
$$

where $\dot{M}$ is the flow rate across the wave: $\dot{M}=\rho(u-\sigma)$. The interest of writing the Rankine-Hugoniot relations as in (18) is that the two last equations are purely thermodynamic. The equation (18b) describes the Rayleigh line in the $(\tau, P)$ plane. Equation (18c) describes the Crussard curve. The very difference with classical shock relations is that the set of the downstream states is not described with the same equation of state as the upstream one. For that sort of wave, we can use the Chapman-Jouguet theory. Let us remind the main points of that theory (see [6] or [4] for the details and the proofs)

Property 1 (Position of the initial state and the Crussard curve). Suppose that the equation of state $(\tau, s) \mapsto P(\tau, s)$ has the following properties

$$
\left(\frac{\partial P}{\partial \tau}\right)_{s}<0 \quad \text { and } \quad\left(\frac{\partial P}{\partial s}\right)_{\tau}>0,
$$

and that the reaction is exothermic

$$
\varepsilon_{1}\left(\tau_{0}, p_{0}\right)<\varepsilon_{0}\left(\tau_{0}, p_{0}\right)
$$

then the point $A_{0}$ corresponding to the upstream state is under the Crussard curve.

In this first property, note that (19a) is always true provided $\gamma$ and $\Gamma$ are both positive (thanks for $(7 \mathrm{c})$ ). We will find in Section 3.1 a condition to ensure the exothermic property $(19 \mathrm{~b})$.

Property 2. Suppose moreover that

$$
\left(\frac{\partial^{2} P}{\partial \tau^{2}}\right)_{s}>0
$$

then the Crussard curve is convex. Hence, the Rayleigh line (18b) and the Crussard curve are crossing in zero or two points.

Note that the Property 2 supposes that the pressure can be differentiated twice, which is not the case in our application, because of the local loss of derivative due to phase transition. Nevertheless, if the properties 1 and 2 hold 


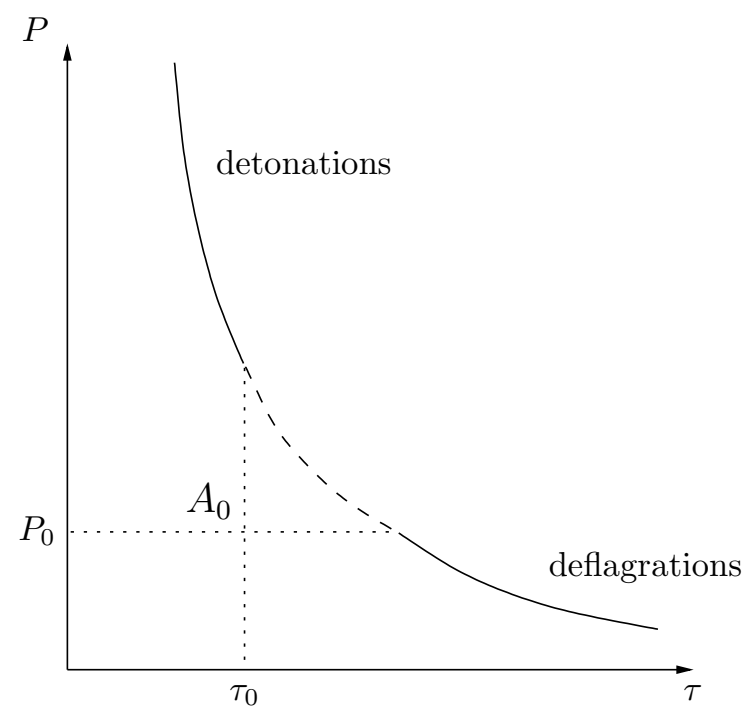

Figure 6: The Crussard curve related to an initial point $\left(\tau_{0}, P_{0}\right)$. The curve is cut into three parts : the upper one is the detonation branch, the lower one is the deflagration branch, and the middle part does not match with the negative slope of the Rayleigh line.

for the equation of state of the downstream states, then the Crussard curve can be schematically drawn as in Figure 6. The Crussard curve is cut into two parts : the upper part is called the detonation branch, and the lower one is the deflagration branch. In the middle part of the curve, $\frac{P_{1}-P_{0}}{\tau_{1}-\tau_{0}}>0$. This does not match with the negative slope of the Rayleigh line (18b).

Each part of the Crussard curve is itself cut into two parts, separated by the tangential point of the Rayleigh line with the Crussard curve (the existence of such tangential point can be shown under some assumption on the asymptotic behavior of equation of state). Both branches are schematically drawn on Figure 7.

Property 3. Along the Crussard curve, the velocity $|v|=|u-\sigma|$ has a local minimum on the CJ-detonation point, and has a local maximum on the CJ-deflagration point. More precisely, we have

$$
\begin{array}{llll}
\text { for a strong detonation }: & \left|v_{0}\right|>c_{0} & \left|v_{1}\right|<c_{1}, \\
\text { for a weak detonation } & : & \left|v_{0}\right|>c_{0} & \left|v_{1}\right|>c_{1}, \\
\text { for a weak deflagration } & : & \left|v_{0}\right|<c_{0} & \left|v_{1}\right|<c_{1}, \\
\text { for a strong deflagration } & : & \left|v_{0}\right|<c_{0} & \left|v_{1}\right|>c_{1} .
\end{array}
$$

This last property is very important because it can allow to know the structure of the half Riemann Problem with a combustion wave provided we know 

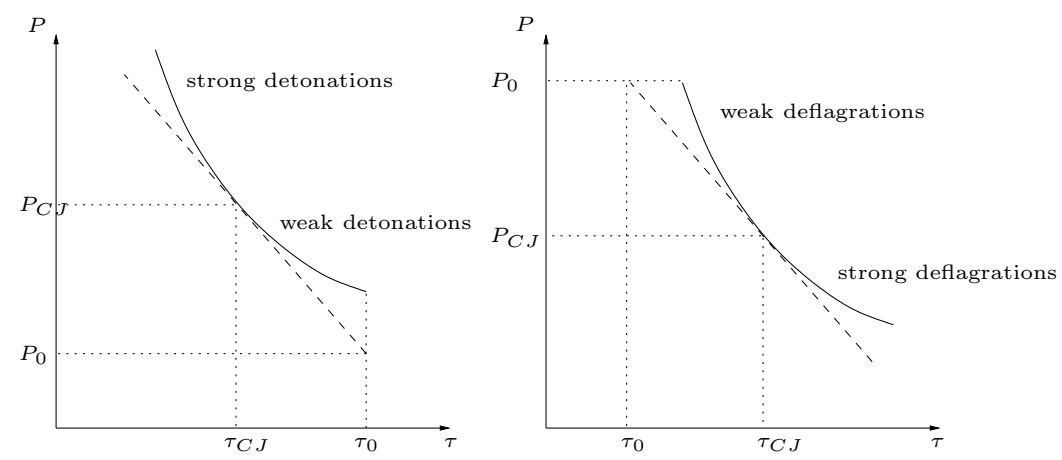

Figure 7: Zoom on the Crussard curve; on the left side, the detonation branch $\left(P \geq P_{0}\right)$ is cut into two parts by the Chapman-Jouguet point. The upper part is the part of the strong detonations, and the lower part is called the part of the weak detonations. On the right side, the deflagration branch $\left(P \leq P_{0}\right)$ is cut into two parts by the Chapman-Jouguet point. The upper part is the part of the weak deflagrations, and the lower part is called the part of the strong deflagrations.

which "family" the combustion wave belongs to. In our case, we are interested in waves which transform a heavy phase into a lighter one. Therefore, we expect that $\tau$ will increase, so that we will concentrate only on the deflagration branch of the Crussard curve.

Thanks for the Property 3, we can give the structure of the Riemann Problem in the case of strong and weak deflagrations. In both cases, from the Property 2 , the deflagration wave is always subsonic relative to the liquid; for example, if the liquid is on the left, we have $\dot{M}>0$, and $u_{0}-c_{0}<\sigma<u_{0}$. For the position of the wave relative to the fields of 1 , we have

- if the wave is a strong deflagration then $\sigma<u_{1}-c_{1}$

- if the wave is a weak deflagration then $u_{1}-c_{1}<\sigma<u_{1}$.

In [4] (p. 230), it is shown that under the assumption that a wave is a deflagration, and that across that wave, the mass fraction of gas always increases, then the reaction is a weak deflagration. We will suppose that we are always in that case in the following.

The structure of the Riemann problem with weak deflagrations is drawn on Figure 8. The problem for deflagrations is that the Lax characteristic criterion is not satisfied (see [6] p. 154), and the Riemann problem cannot be solved only with the classical relations across the sonic wave and the Rankine-Hugoniot relations across the subsonic wave. There remains one indeterminate. The supplementary relation needed is often called "the kinetic closure". 


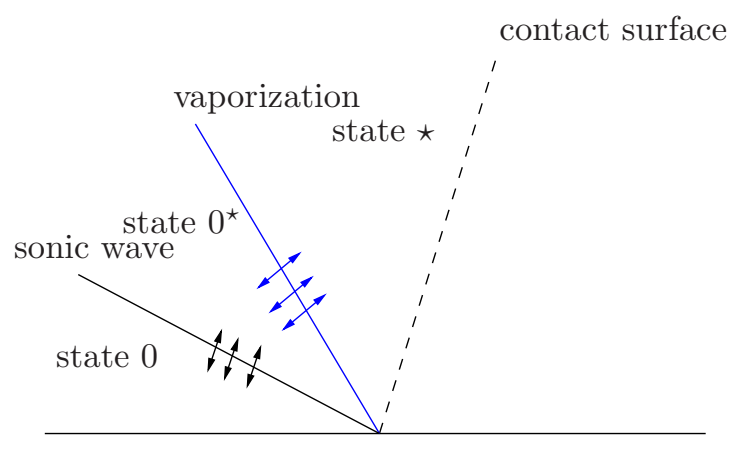

Figure 8: Structure of the half Riemann problem : the state 0 is linked with the state $0^{\star}$ by a forerunner sonic wave (rarefaction wave or shock). Then the state $0^{\star}$ and the state $\star$ are linked with a deflagration wave. Eventually, there is a contact discontinuity.

\section{Application to the solution of the Riemann problem with vaporization}

\subsection{Useful verifications for the use of CJ theory}

In that section, we check whether the inequalities needed for applying the Chapman-Jouguet theory hold.

Theorem 3. 1. If both equations of state are convex, and if $\frac{\mathrm{d} P_{\text {sat }}}{\mathrm{d} T}>0$, then inequalities (19a) hold.

2. If $(P, \tau)$ lie in the saturation dome and under the condition

$$
\frac{\gamma}{\Gamma}-\frac{T}{P} \frac{\mathrm{d} P_{\text {sat }}}{\mathrm{d} T}>0
$$

the inequality (19b) holds.

Proof. According to the identity (7c), it is sufficient to have $\gamma$ and $\Gamma>0$. This is supposed for pure fluids, and this is ensured for mixture equation of state if $\frac{\mathrm{d} P_{\text {sat }}}{\mathrm{d} T}>0$, so that (19a) holds.

Let us now check if the inequality $(19 \mathrm{~b})$ is ensured. We suppose that $(P, \tau)$ lie in the saturation dome, so that the corresponding equilibrium downstream state is a mixture :

$$
\varepsilon_{1}(\tau, P)=\varepsilon_{m}(\tau, P)=y_{l} \varepsilon_{l}\left(P, \tau_{l}(P)\right)+\left(1-y_{l}\right) \varepsilon_{v}\left(P, \tau_{v}(P)\right),
$$

and we want to know if $\varepsilon_{1}(P, \tau)-\varepsilon_{0}(P, \tau)<0$, the state 0 being of course described by the liquid equation of state. For that, we denote by

$$
\delta \varepsilon\left(y_{l}\right)=y_{l} \varepsilon_{l}\left(P, \tau_{l}(P)\right)+\left(1-y_{l}\right) \varepsilon_{v}\left(P, \tau_{v}(P)\right)-\varepsilon_{l}\left(P, y_{l} \tau_{l}(P)+\left(1-y_{l}\right) \tau_{v}(P)\right),
$$


and we immediately see that $\delta \varepsilon(1)=0$. It remains to show that $\delta \varepsilon$ is an increasing function

$$
\begin{aligned}
\frac{\mathrm{d} \delta \varepsilon}{\mathrm{d} y_{l}}\left(y_{l}\right)=\varepsilon_{l} & \left(P, \tau_{l}(P)\right)-\varepsilon_{v}\left(P, \tau_{v}(P)\right) \\
& -\left(\tau_{l}(P)-\tau_{v}(P)\right)\left(\frac{\partial \varepsilon_{l}}{\partial \tau}\right)_{P}\left(P, y_{l} \tau_{l}(P)+\left(1-y_{l}\right) \tau_{v}(P)\right) .
\end{aligned}
$$

Integration of the identity $\mathrm{d} \varepsilon+P \mathrm{~d} \tau=T \mathrm{~d} s$ across the saturation dome leads to

$$
\varepsilon_{l}\left(P, \tau_{l}(P)\right)-\varepsilon_{v}\left(P, \tau_{v}(P)\right)+P\left(\tau_{l}(P)-\tau_{v}(P)\right)=T\left(s_{l}(P)-s_{v}(P)\right),
$$

so that

$$
\begin{aligned}
\frac{\mathrm{d} \delta \varepsilon}{\mathrm{d} y_{l}}\left(y_{l}\right)=- & P\left(\tau_{l}(P)-\tau_{v}(P)\right)+T\left(s_{l}(P)-s_{v}(P)\right) \\
& \quad\left(\tau_{l}(P)-\tau_{v}(P)\right)\left(\frac{\partial \varepsilon_{l}}{\partial \tau}\right)_{P}\left(P, y_{l} \tau_{l}(P)+\left(1-y_{l}\right) \tau_{v}(P)\right)
\end{aligned},
$$

which can be cast into the following form, thanks for (5) and (7f)

$$
\frac{\mathrm{d} \delta \varepsilon}{\mathrm{d} y_{l}}\left(y_{l}\right)=P\left(\tau_{v}-\tau_{l}\right)\left(\frac{\gamma}{\Gamma}-\frac{T}{P} \frac{\mathrm{d} P_{\text {sat }}}{\mathrm{d} T}\right) .
$$

As $P>0, \tau_{v}-\tau_{l}>$, and as $(20), \delta \varepsilon$ increases, so that $\delta \varepsilon \leq \delta \varepsilon(1)=0$. Thus, (19b) holds.

\section{Remark 1}

Supposing that $(\tau, P)$ is always in the saturation dome is not a strong assumption. Indeed, as the upstream state is few compressible, its specific volume cannot increase a lot across a sonic wave, and it is likely that a metastable liquid with a specific volume equal to the one of a gas at equilibrium cannot exist, except just near the critical point.

\section{Remark 2}

The condition (20) holds at least in the two following framework

1. The terms $\frac{\gamma}{\Gamma}$ and $\frac{T}{P} \frac{\mathrm{d} P_{\text {sat }}}{\mathrm{d} T}$ can easily be compared near the saturation curve. Indeed, if we use (7b) with saturated variables, we find

$$
\frac{T^{2}\left(\gamma g-\Gamma^{2}\right)}{P \tau} \frac{\mathrm{d} s_{l}}{\mathrm{~d} T}=\frac{\gamma}{\Gamma}-\frac{T}{P} \frac{\mathrm{d} P_{\text {sat }}}{\mathrm{d} T}
$$

so that we have $\frac{\mathrm{d} \delta \varepsilon}{\mathrm{d} T}(1)=\left(\tau_{v}-\tau_{l}\right) \frac{T^{2}}{\tau} \frac{\gamma g-\Gamma^{2}}{\Gamma} \frac{\mathrm{d} s_{l}}{\mathrm{~d} T}$.

Thus, if the liquid saturation curve is regular (which is always the case) then $\frac{\mathrm{d} s_{l}}{\mathrm{~d} T}>0$, so that the condition (20) is ensured. 
2. For simple model, as perfect gas or stiffened gas, we have

$$
\frac{\gamma}{\Gamma}=\frac{\bar{\gamma}\left(1+\frac{P^{\infty}}{P}\right)}{\bar{\gamma}-1}
$$

so that $\frac{\gamma}{\Gamma}$ does not depend on the specific volume. Thus, equality (21) holds for any $\tau$, so that the condition (20) always holds.

\section{Remark 3}

The same calculations can be made for liquefaction. Then we find, near the vapor saturation curve

$$
\frac{\mathrm{d} \delta \varepsilon}{\mathrm{d} T}(1)=\left(\tau_{l}-\tau_{v}\right) \frac{T^{2}}{\tau} \frac{\gamma g-\Gamma^{2}}{\Gamma} \frac{\mathrm{d} s_{v}}{\mathrm{~d} T} .
$$

Thus, if the fluid is regular then $\frac{\mathrm{d} s_{v}}{\mathrm{~d} T}<0$, and as $\tau_{l}-\tau_{v}<0$ then $\frac{\mathrm{d} \delta \varepsilon}{\mathrm{d} T}(1)>0$, so that locally we have $\varepsilon_{m}-\varepsilon_{v}<0$, and the Chapman-Jouguet theory can be used. If the fluid is retrograde, then we find that locally $\varepsilon_{m}-\varepsilon_{v}>0$ and the Chapman-Jouguet theory may be used, but by exchanging the upstream and the downstream state. Note that the Hugoniot curves might enter the saturation dome only in the retrograde case.

The Chapman-Jouguet theory relies also a lot on the convexity properties of the Crussard curve (see Property 2 of Section 2), which is ensured if the fundamental derivative $\mathscr{G}$ is positive. Nevertheless, even if we suppose that the liquid and the gas equation of state have a positive fundamental derivative, the mixture equation of state can have a negative fundamental derivative, as it was shown numerically in Section 1.8. This non positivity of the fundamental derivative can lead to a wrong behavior of the Crussard curve as shown in Figure 9: the CJ points do not exist any more and all the undercompressive downstream states are strong deflagrations. If the sign of the fundamental derivative changed many times along the Crussard curve, we could expect to observe several CJ points. From now, we suppose that $\mathscr{G}>0$.

\subsection{Entropy growth criterion}

As the particles are crossing the front from the liquid area to a mixture or pure phase area, we have to check whether the entropy growth criterion is ensured, i.e. if the entropy of the downstream state (gas or mixture) is greater than the entropy of the upstream state (liquid). We first prove the following theorem.

Theorem 4. Let $s=s_{0}$ a liquid isentrope that crosses the liquid saturation curve. To any metastable point $\left(\tau_{0}, P_{0}\right)$ on that isentrope, we map the point 


\section{Pressure $(\mathrm{Pa})$}

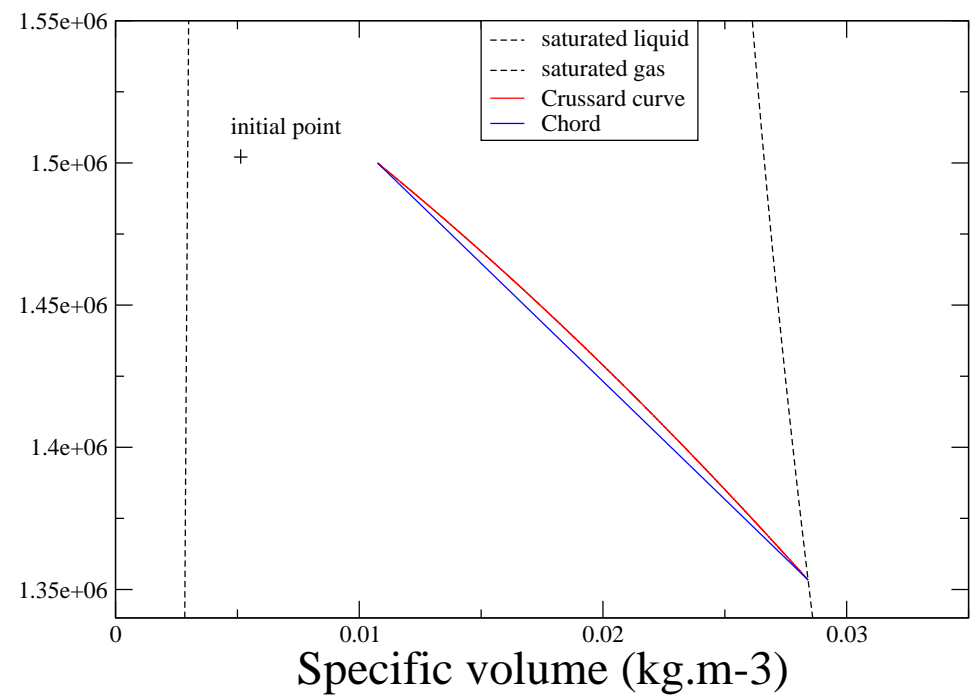

Figure 9: Wrong behavior of the mixture Crussard curve if the condition $\mathscr{G}>0$ is violated. We notice that the Crussard curve is concave which induces no existence of any CJ point. For a more complicated couple of equation of state, we could expect to observe two or three tangential points if the sign of $\mathscr{G}$ changed two or three times along the Crussard curve. 


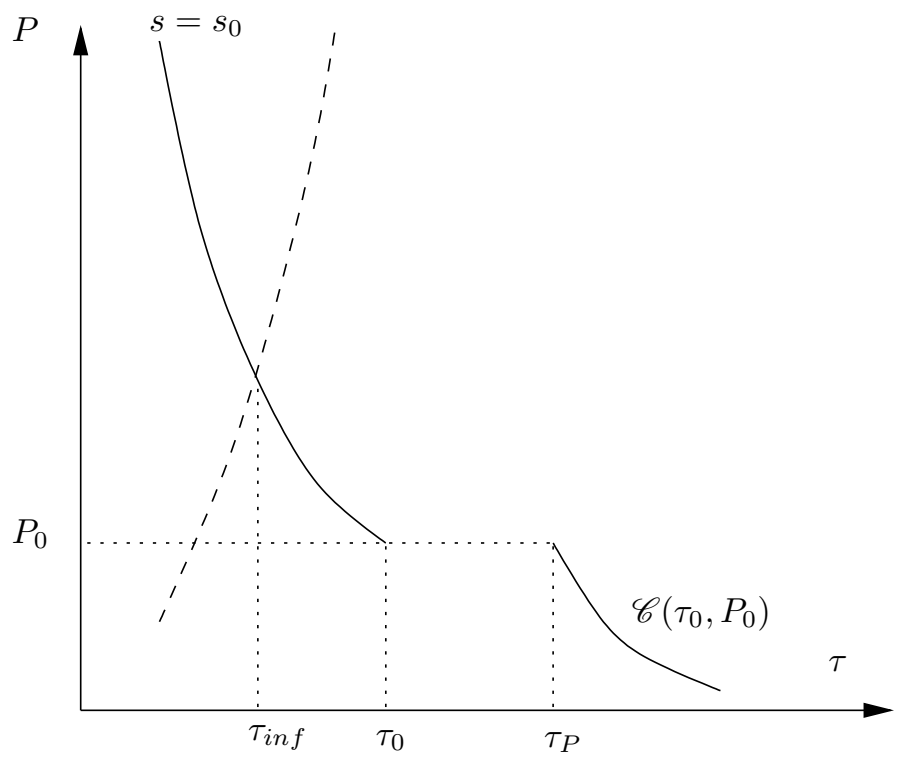

Figure 10: Entropy growth criterion. To any point $\left(\tau_{0}, P_{0}\right)$ on a given isentrope $s=s_{0}$, we associate the point on the Crussard curve $\left(\tau_{P}, P_{0}\right)$. The liquid saturation curve is drawn on dashed lines. When $\tau_{0}$ is on the liquid saturation curve, we have $\tau_{P}=\tau_{0}$, so that $s_{P}=s_{0}$. Thus, to show that $s_{P} \geq s_{0}$, we only have to prove that entropy of the point $P$ grows when $\tau_{0}$ increases.

$\left(\tau_{P}, P_{0}\right)$, point of constant pressure deflagration (see Figure 10). If we suppose that

$$
\forall\left(\tau_{0}, P_{0}\right) \quad \gamma\left(\tau_{0}, P_{0}\right)>\gamma\left(\tau_{P}, P_{0}\right)
$$

then $s\left(\tau_{P}, P_{0}\right)>s_{0}$.

Proof. To any point $\left(\tau_{0}, P_{0}\right)$ on this isentrope, we associate the point $\left(\tau_{P}, P_{0}\right)$, point of constant pressure deflagration (see Figure 10). For more conveniency in the notations, we suppose that $\left(\tau_{P}, P_{0}\right)$ is a mixture state (i.e. all the linked quantities have $m) . \tau_{P}$ is defined by the implicit equation

$$
\varepsilon_{m}\left(\tau_{P}, P_{0}\right)-\varepsilon_{l}\left(\tau_{0}, P_{0}\right)+P_{0}\left(\tau_{P}-\tau_{0}\right)=0
$$

Differentiation of (23) with respect to $\tau_{P}$ is equal to $\frac{\gamma_{m}}{\Gamma_{m}}$, which never vanishes, so that according to the implicit function theorem, $\tau_{P}$ is a $\mathscr{C}^{1}$ function of $\tau_{0}$ 
and $P_{0}$. Moreover, we can calculate its derivative with respect to $\tau_{0}$ and $P_{0}$ :

$$
\left\{\begin{array}{l}
\left(\frac{\partial \tau_{P}}{\partial \tau_{0}}\right)_{P_{0}}=\frac{\gamma_{l}}{\gamma_{m}} \frac{\Gamma_{m}}{\Gamma_{l}} \\
\left(\frac{\partial \tau_{P}}{\partial P_{0}}\right)_{\tau_{0}}=\frac{\tau_{0}}{\gamma_{m} P_{0}}\left(\frac{\Gamma_{m}\left(\Gamma_{l}+1\right)}{\Gamma_{l}}-\frac{\tau_{P}}{\tau_{0}}\left(\Gamma_{m}+1\right)\right)
\end{array}\right.
$$

Besides, as we supposed that the points $\left(P_{0}, \tau_{0}\right)$ belong to the same isentrope, $P_{0}$ is actually a function of $\tau_{0}$ with $\frac{\mathrm{d} P_{0}}{\mathrm{~d} \tau_{0}}=-\frac{\gamma_{l} P_{0}}{\tau_{0}}$, so that $\tau_{P}$ is a function of the only variable $\tau_{0}$ and

$$
\begin{aligned}
\frac{\mathrm{d} \tau_{P}}{\mathrm{~d} \tau_{0}} & =\left(\frac{\partial \tau_{P}}{\partial \tau_{0}}\right)_{P_{0}}+\frac{\mathrm{d} P_{0}}{\mathrm{~d} \tau_{0}}\left(\frac{\partial \tau_{P}}{\partial P_{0}}\right)_{\tau_{0}} . \\
& =\frac{\gamma_{l}}{\gamma_{m}}\left(-\Gamma_{m}+\frac{\tau_{P}}{\tau_{0}}\left(\Gamma_{m}+1\right)\right)
\end{aligned} .
$$

Now, we calculate the entropy variation of the point $\tau_{P}$ when the point $\left(P_{0}, \tau_{0}\right)$ follows the isentrope $s=s_{0}$

$$
\begin{aligned}
\frac{\mathrm{d} s}{\mathrm{~d} \tau_{0}} & =\frac{\mathrm{d} \tau_{P}}{\mathrm{~d} \tau_{0}}\left(\frac{\partial s}{\partial \tau}\right)_{P}+\frac{\mathrm{d} P_{0}}{\mathrm{~d} \tau_{0}}\left(\frac{\partial s}{\partial P}\right)_{\tau} \\
& =\frac{\gamma_{m} P_{0}}{T \Gamma_{m}}\left(\frac{\gamma_{l} \Gamma_{m}}{\gamma_{m}}\left(\frac{\tau_{P}}{\tau_{0}}-1\right)+\frac{\tau_{P}}{\tau_{0}}\left(\frac{\gamma_{l}}{\gamma_{m}}-1\right)\right) .
\end{aligned}
$$

According to the hypothesis (22), $\gamma_{l}>\gamma_{m}$. Moreover as we have $\tau_{P}-\tau_{0}>0, s$ is an increasing function of $\tau_{0}$. Furthermore, in the limit of no overheating, we have

$$
\lim _{\tau_{0} \rightarrow \tau_{\text {inf }}} \tau_{P}\left(\tau_{0}, P_{0}\right)=\tau_{\text {inf }},
$$

where $\tau_{\text {inf }}$ is the crossing point of the isentrope $s=s_{0}$ with the saturation curve. Thus $\lim _{\tau_{0} \rightarrow \tau_{\text {inf }}} s\left(\tau_{P}, P_{0}\right)=s_{0}$. As a conclusion

$$
\forall \tau_{0} \geq \tau_{\text {inf }} \quad s\left(\tau_{P}, P_{0}\right) \geq s_{0},
$$

which ends the proof.

Remark (About the hypothesis (22))

1. We know that near the saturation curve, we have $\gamma_{l}>\gamma_{m}$. For actual data, we have $\gamma_{l} \gg \gamma_{m}$. So that we can suppose that any $\gamma$ is greater than any $\gamma_{m}$.

2. $\gamma_{l}>\gamma_{v}$ just means that the liquid phase is very much less compressible than the gas phase (see e.g. [22] chapter XI).

Given an initial point, we know that the entropy grows from the constant pressure point to the Chapman-Jouguet point, so that if the entropy growth criterion holds for the constant pressure deflagration point, it holds for all the downstream states between the constant pressure deflagration point and the Chapman-Jouguet point, i.e. 
Corollary 1. Under the same hypothesis as the Theorem 4, the entropy growth criterion holds for all the weak deflagration points.

\subsection{Behavior of the Crussard curve near the gas satura- tion curve}

In Section 1.5, the behavior of the isentropes near the saturation curves was studied. The difference of the differential behavior of the pure phase and the mixture equation of state induced kinks in isentropes. Now, we want to study the behavior of the Crussard curve when it crosses the vapor saturation curve. It is more difficult than the study of the isentrope, because the Crussard curve does not depend only on the local variables, but also on the starting point $\left(\tau_{0}, P_{0}\right)$.

\subsubsection{General study}

We denote by $C$ the point in which the Crussard curve crosses the saturation curve, and by

$$
\zeta=-\frac{\tau}{P} \frac{\mathrm{d} P}{\mathrm{~d} \tau} \mathscr{C}^{\prime},
$$

the adimensioned slope of the Crussard curve.

The first thing we will prove for the behavior of the Crussard curve near the saturation curve, is that it can be parameterized by $\tau$, under some conditions

Theorem 5. If all the equations of state are convex and if $\Gamma>0$, then $\zeta>0$. With the same hypothesis, the Crussard curve can be parameterized by $\tau$, even near the saturation curve.

Proof. As proved in [12, page 101], we have

$$
\zeta=\frac{\frac{\gamma}{\Gamma}-\frac{\Delta P}{2 P}}{\frac{1}{\Gamma}+\frac{\Delta \tau}{2 \tau}}
$$

Across a deflagration wave, we have $\Delta \tau>0$ and $\Delta P<0$. Moreover, we proved that $\Gamma_{m}>0$, and we suppose that $\Gamma>0$. The conditions $\gamma>0$ and $\gamma_{m}>0$ were already supposed to ensure the convexity of the specific energy. Then $\zeta>0$. If we combine (24) with the identities near the saturation boundary of Section 1.5 , we get

$$
\frac{\xi-\zeta}{\Gamma}\left(1+\Gamma \frac{\Delta \tau}{2 \tau}\right)=\frac{\xi-\zeta_{m}}{\Gamma_{m}}\left(1+\Gamma_{m} \frac{\Delta \tau}{2 \tau}\right)
$$

Across a deflagration, we have $\Delta \tau>0$. Then

$$
\frac{\xi-\zeta}{\xi-\zeta_{m}}>0
$$


which means that the Crussard curve, near a boundary, can be parameterized by $\tau$. As $\zeta>0$, the Crussard curve is a diffeomorphism of $\tau$ in each side of the saturation curve. As the Crussard curve can locally parameterize the Crussard curve near a boundary, we conclude that the Crussard curve is a homeomorphism of $\tau$.

Remark As the Crussard curve is a decreasing homeomorphism in $\tau$, the point of constant pressure deflagration is uniquely defined.

To be more precise on the relative behavior of the isentropes, the Crussard curve, and the saturation curve, we will prove that

Theorem 6. The relative behavior of the isentropes and the Crussard curves, which gives the nature of the deflagration on each side of the saturation curve follows the alternative

- if $\gamma>\xi$ then

- either $\xi \geq \zeta$, then the deflagration is weak on both sides of the saturation curve,

- or $\xi<\zeta$, then point $C$ cannot be a weak deflagration simultaneously on both sides of the saturation curve.

- if $\gamma<\xi$ then

- either $\xi \leq \zeta$, then the deflagration is strong on both of the sides of the saturation curve

- or $\xi>\zeta$, then the deflgration cannot be simultaneously strong on the mixture side, and weak on the pure phase side.

Proof. Equation (25) can be rewritten as

$$
\frac{\xi-\zeta}{\xi-\zeta_{m}}=\frac{\frac{1}{\Gamma_{m}}+\frac{\Delta \tau}{2 \tau}}{\frac{1}{\Gamma}+\frac{\Delta \tau}{2 \tau}}
$$

so that the discontinuity in the slope of the Crussard curve is directly linked with the sign of $\Gamma_{m}-\Gamma$ (we recall that $\Gamma_{m}>0$ and that $\Gamma$ has the same sign as $\Gamma_{m}$ near the saturation curves). Equation (14) induces a separation into the following cases

- $\gamma>\xi$

If $\gamma>\xi$ then we also have $\gamma_{m}>\xi$. As $\gamma_{m}<\gamma$, we have $\frac{\gamma_{m}-\xi}{\gamma-\xi} \leq 1$, so that $\Gamma_{m} \leq \Gamma$. Therefore

$$
\frac{\xi-\zeta}{\xi-\zeta_{m}} \geq 1
$$




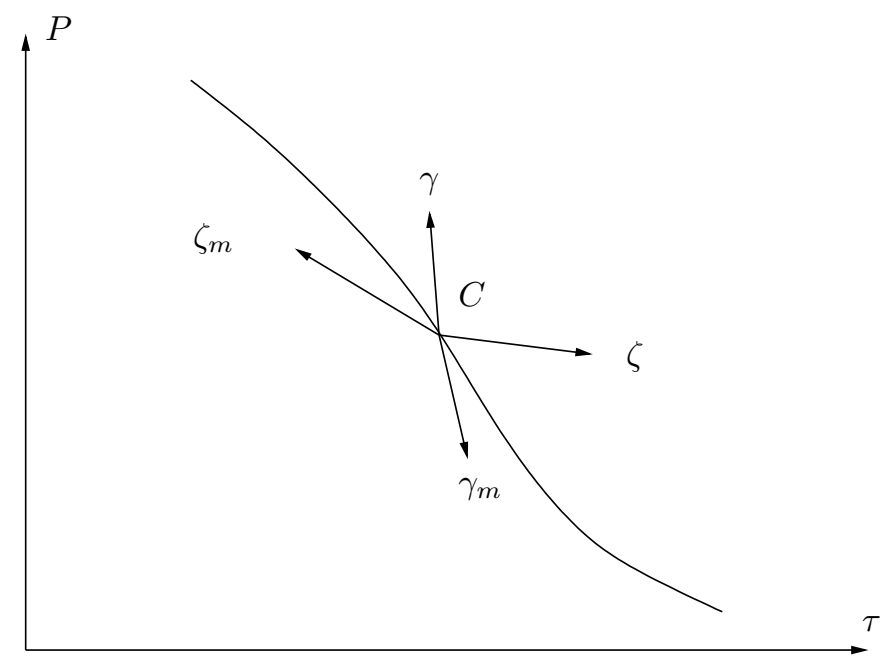

Figure 11: Qualitative relative behavior of the isentrope and of the Crussard curve when they cross the vapor saturation curve in the case 1. Arrows represent half tangent of the Crussard curve $(\zeta)$ and of the isentrope $(\gamma)$

Suppose first that $\xi-\zeta \geq 0$. Then $\xi-\zeta \geq \xi-\zeta_{m}$ so that $\zeta \leq \zeta_{m} \leq \xi$. In that case, as shown on the Figure 11, the relative behavior of the isentrope and the Crussard curve show that in both sides of the saturation curve, the downstream state is a weak deflagration (see Figure 11). In that case, we have $\zeta \leq \zeta_{m} \leq \xi \leq \gamma_{m} \leq \gamma$.

Suppose now that $\xi-\zeta \leq 0$. Then we have $\xi \leq \zeta_{m} \leq \zeta$. The nature of the deflagration is given by the relative position of the slope of the Crussard curve and the Rayleigh line, so that on the point saturation curve, there are three cases (see Figure 12):

- If the Rayleigh line has a lower slope than both of the slopes of the Crussard curve, then the two parts match with strong deflagrations. Thus, we have $\gamma_{m} \leq \zeta_{m}$ and $\gamma \leq \zeta$ (see Figure 12, case $(a)$ ).

- If the slope of the Rayleigh line is between the slopes of the Crussard curve, then the mixture Crussard curve matches with strong deflagrations whereas the pure phase Crussard curve matches with weak deflagrations. In that case, we have $\gamma_{m} \leq \zeta_{m}$ and $\gamma \geq \zeta$ (see Figure 12 , case $(b))$. In that case we have $\xi \leq \zeta_{m} \leq \gamma_{m} \leq \gamma \leq \zeta$.

- If the Rayleigh line has a greater slope than both of the slopes of the Crussard curve then the point $C$ is a weak deflagration with respect to the pure and the mixture Crussard curve. Therefore, we have $\gamma_{m} \geq \zeta_{m}$ and $\gamma \geq \zeta$ (see Figure 12 , case $(c)$ ). 


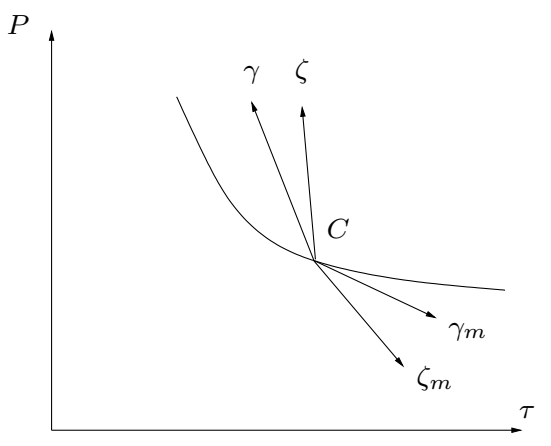

(a)

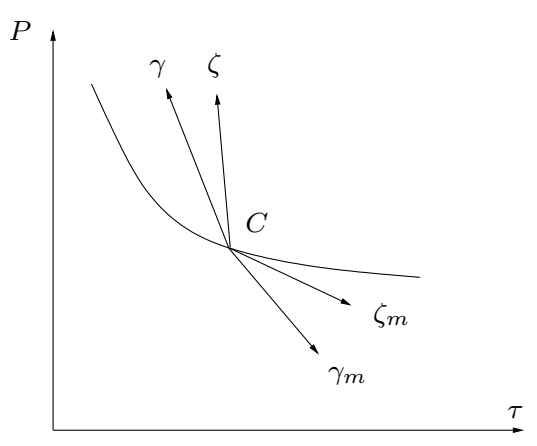

(b)

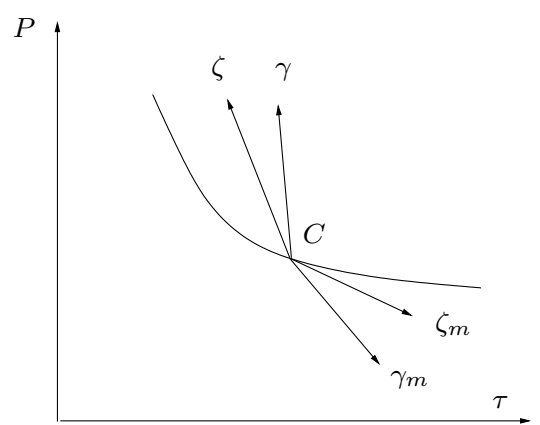

(c)

Figure 12: Qualitative relative behavior of the isentrope and of the Crussard curve when they cross the vapor saturation curve, case 2 . Arrows represent half tangent of the Crussard curve $(\zeta)$ and of the isentrope $(\gamma)$. 


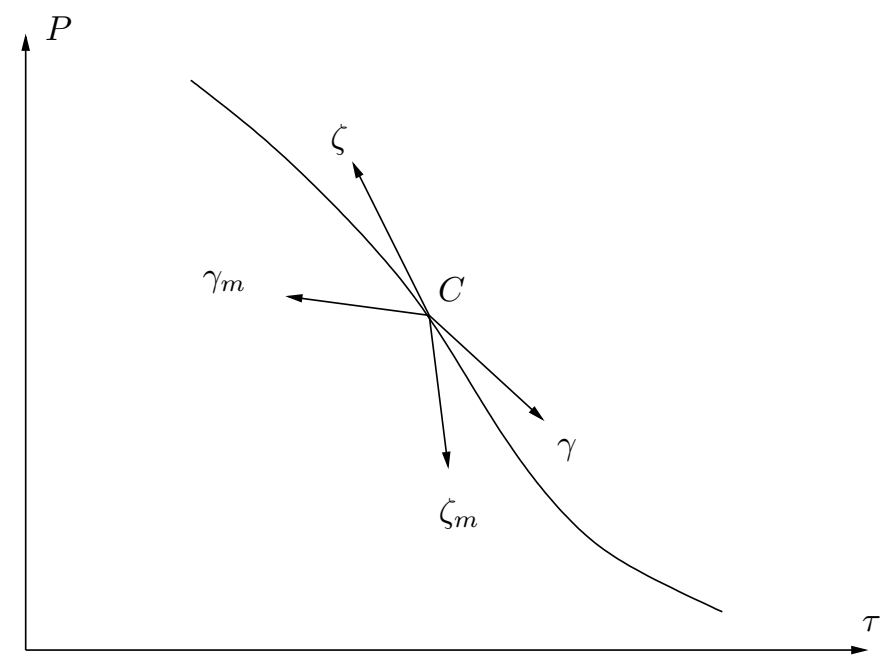

Figure 13: Qualitative relative behavior of the isentrope and of the Crussard curve when they cross the vapor saturation curve, case 3. Arrows represent half tangent of the Crussard curve $(\zeta)$ and of the isentrope $(\gamma)$.

- $\gamma<\xi$

If $\gamma<\xi$ then we also have $\gamma_{m}<\xi$. As we know that $\gamma_{m} \leq \gamma$, we have $\frac{\gamma_{m}-\xi}{\gamma-\xi} \geq 1$, so that $\Gamma_{m} \geq \Gamma$ (thanks for equation (14)). Therefore

$$
\frac{\xi-\zeta}{\xi-\zeta_{m}} \leq 1
$$

We suppose first that $\xi-\zeta \leq 0$. Then we immediately have $\zeta \leq \zeta_{m}$. Thus, we have $\gamma_{m} \leq \zeta_{m}$ and $\gamma \leq \zeta$, so that point $C$ matches on both sides of the Crussard curve with strong deflagrations (see Figure 13). In that case we have $\zeta_{m} \leq \zeta \leq \xi \leq \gamma \leq \gamma_{m}$.

We suppose now that $\xi-\zeta \geq 0$. Then we have $\zeta_{m} \leq \zeta \leq \xi$. The nature of the deflagration is given by the relative position of the slope of the Crussard curve and the Rayleigh line, so that three cases may happen (see Figure 14):

- If the Rayleigh line has a lower slope than the slopes on both sides of the Crussard curve in $C$, then the point $C$ is a weak deflagration with respect to the mixture and the pure phase Crussard curve. We have then $\gamma \leq \zeta$ and $\gamma_{m} \leq \zeta_{m}$ (see Figure 14, case $(a)$ ).

- If the slope of the Rayleigh line is between the slopes on each side of the Crussard curve, then point $C$ is a strong deflagration for the mixture Crussard curve, and a weak deflagration for the pure phase 


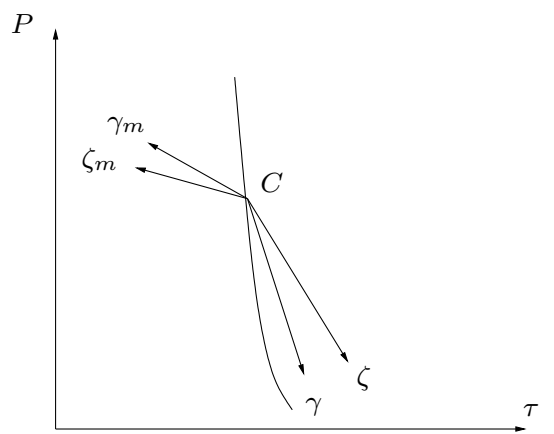

(a)

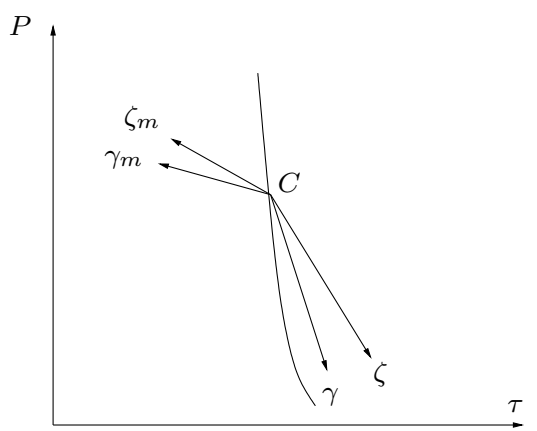

(b)

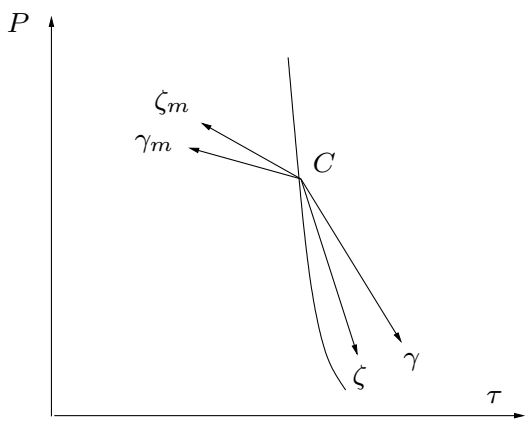

(c)

Figure 14: Qualitative relative behavior of the isentrope and of the Crussard curve when they cross the vapor saturation curve, case 4 . Arrows represent half tangent of the Crussard curve $(\zeta)$ and of the isentrope $(\gamma)$.

Crussard curve (see Figure 14, case $(b)$ ). In that case we have $\gamma_{m} \leq$ $\zeta_{m} \leq \zeta \leq \gamma \leq \xi$.

- If the Rayleigh line has a lower slope than both of the slopes of the Crussard curve then the point $C$ is a strong deflagration with respect to the pure and the mixture Crussard curve. Therefore, we have $\gamma_{m} \geq \zeta_{m}$ and $\gamma \geq \zeta$ (see Figure 14, case $(c)$ ).

This ends the proof.

\subsubsection{Ill-posedness of the Chapman-Jouguet closure}

The following result comes immediately from the Theorem 6

Corollary 2. Let $\left(P_{0}, \tau_{0}, u_{0}\right)$ be an initial state of liquid at thermodynamic equilibrium, such that the isentrope $\mathscr{C}_{s}$ coming from this point enters the saturation dome. If $\left(P_{0}^{\star}, \tau_{0}^{\star}\right)$ is a point in $\mathscr{C}_{s}$, we build $\left(P^{\star}, \tau^{\star}\right)$ in the following way

- if $\left(P_{0}^{\star}, \tau_{0}^{\star}\right)$ is not in the saturation dome then $P^{\star}=P_{0}^{\star}$, and $\tau^{\star}=\tau_{0}^{\star}$, 


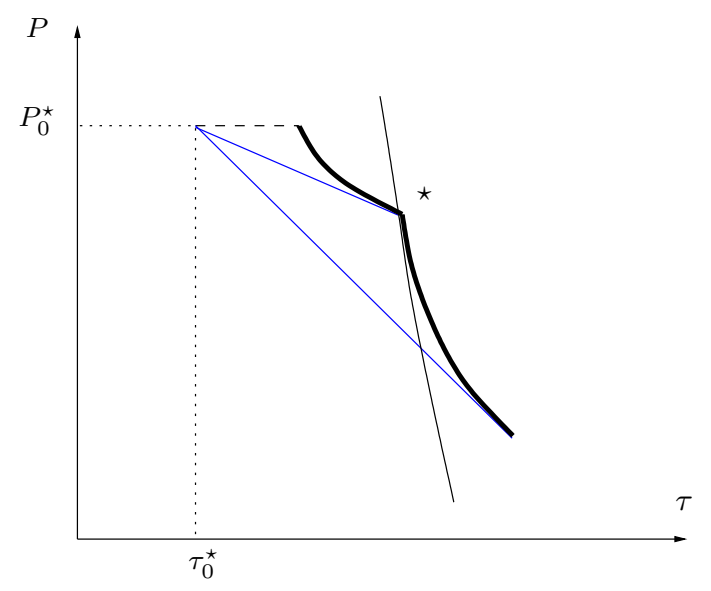

Figure 15: Qualitative behavior of the Rayleigh line and the Crussard curve when the Crussard curve crosses the saturation curve on a mixture ChapmanJouguet point. As $\zeta_{m} \leq \zeta$, and as the Rayleigh line is tangential to the mixture Crussard curve, we are in the case (4.c) of the proof of Theorem 6. As a consequence, the pure phase side matches with a weak deflagrtion too, so that there exist another Chapman-Jouguet point.

- if $\left(P_{0}^{\star}, \tau_{0}^{\star}\right)$ is in the saturation dome, then it is linked with $\left(P^{\star}, \tau^{\star}\right)$ with a Chapman-Jouguet deflagration.

If $\left(P^{\star}, \tau^{\star}\right)$ can reach the pure gas phase, then the curve $\left(P^{\star}, \tau^{\star}\right)$ is discontinuous.

Proof. We suppose that the set described is continuous. As $\left(P^{\star}, \tau^{\star}\right)$ can reach the saturation dome, it crosses the gas saturation curve on a point $\left(P_{c}, \tau_{c}\right)$. As it is a Chapman-Jouguet point, we have $\gamma_{m}=\zeta_{m}$, so that we are in case 4 of Figure 14. The case 4. $(c)$ is excluded because the Rayleigh line is tangential with the Crussard curve, so that the slope of the Rayleigh line is greater than the slope of the Crussard curve in the pure phase side. Thus, the point $\star$ matches with a weak deflagration with respect to the pure phase Crussard curve. As a consequence, the Crussard curve has another Chapman-Jouguet point that lies in the pure phase area (see Figure 15), so that the curve $\mathscr{C}_{C J}$ has already a branch in the pure gas area.

Eventually, we can state the following theorem

Theorem 7. With the same hypothesis of Corollary 2, if we model the vaporization wave by a Chapman-Jouguet deflagration, then the resulting solution of the Riemann problem is ill-posed in the $L^{1}$ sense: the solution does not depend continuously on the initial state.

Proof. We fix a point for $x<0$ in the liquid area for which the conditions of Corollary 2 hold, and we suppose that on the right, there is some gas. The 


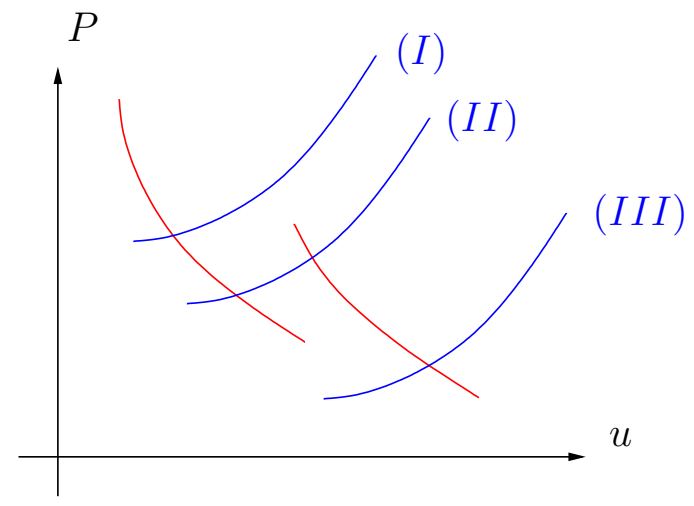

Figure 16: In red, the wave curve of the liquid side. In blue, different wave curves for the gas side, depending on the initial state. In the case $(I)$, the gas wave curve intersects the liquid one in one mixture point. In the case $(I I)$, the gas wave curve intersects the liquid one in the two branches: one mixture and one pure gas point. In the case $(I I I)$, the gas wave curve intersects the liquid one only on the pure phase branch.

Riemann problem is composed (from the left to the right) of a sonic wave, a vaporization wave (if the intermediate state is metastable), a contact discontinuity (across which $P$ and $u$ are constant), and a sonic wave in the gas side. As it is done usually [6], to solve the Rieman problem, we intersect the wave curve of the downstream state (sonic wave and maybe followed by a CJ vaporization) of the left side with the wave curve of the sonic wave of the right side, in the plane $(P, u)$. Corollary 2 says that the wave curve of the left side is composed of (at least) two branches (see Figure 16). So that the gas wave curve intersects the liquid wave curve either in one mixture point (case $(I)$ ), or in two points (case $(I I)$ ), or in one pure gas point (case $(I I I)$ ). Existence of case $(I)$ and case (III) implies that we must jump from the mixture to the gas branch of the liquid wave curve. But jumping from one branch to the other means that we change a lot the vaporizes state (so the $L_{l o c}^{1}$ norm too), but by changing few the initial state.

We finish this paper by drawing the curve $\mathscr{C}_{C J}$ described in Corollary 2 for the models of equation of state of Section 1.

Example 1 : two perfect gas equation of state

As an example, we take the model with two perfect gas. As we said before, this model enables to make all the calculation, because the mixture equation of state is explicit.

\section{- Mixture CJ-point}


In the case when the downstream state is a mixture, the equation of the Crussard curve is the following

$$
\frac{P \tau_{2}}{\Gamma_{2}}-\frac{P_{0} \tau_{0}}{\Gamma_{2}}+\frac{1}{2}\left(P+P_{0}\right)\left(\tau-\tau_{0}\right)=0
$$

which gives an expression of $\tau$ as a function of $P: \tau=\tau_{0}-\frac{2\left(P \tau_{2}-P_{0} \tau_{0}\right)}{\Gamma_{2}\left(P+P_{0}\right)}$. The CJ-point is such that $\frac{\mathrm{d} \tau}{\mathrm{d} P}\left(P_{C J}\right)=\frac{\tau-\tau_{0}}{P-P_{0}}$, so that we find the following equation for $P_{C J}$

$$
\left(\frac{P}{P_{0}}\right)^{2}-2 \frac{\tau}{\tau_{0}} \frac{P}{P_{0}}+1=0
$$

whose undercompressive solution is

$$
P_{C J}=P_{0}\left(\frac{\tau_{0}}{\tau_{2}}-\sqrt{\left(\frac{\tau_{0}}{\tau_{2}}\right)^{2}-1}\right)
$$

$\tau_{C J}$ is then given by

$$
\tau_{C J}=\tau_{2}\left(\frac{\tau_{0}}{\tau_{2}}+\frac{2 \sqrt{\frac{\tau_{0}}{\tau_{2}}-1}}{\Gamma_{2}\left(\frac{\tau_{0}}{\tau_{2}}+1-\sqrt{\left(\frac{\tau_{0}}{\tau_{2}}\right)^{2}-1}\right)}\right) .
$$

Of course, this point can be chosen only when the mixture is stable, that means when $\tau_{C J} \leq \tau_{1}$.

\section{- Vapor CJ-point}

The equation of the Crussard curve is then

$$
\frac{\tau P}{\Gamma_{1}}-\frac{\tau_{0} P_{0}}{\Gamma_{2}}+\frac{1}{2}\left(P+P_{0}\right)\left(\tau-\tau_{0}\right)=0 .
$$

As in [10], we first calculate the point of constant specific volume detonation, i.e. the downstream state such as $\tau=\tau_{0}: P_{\tau}=\frac{\Gamma_{1}}{\Gamma_{2}} P_{0}$. If we take the calculations of [10], we get

$$
P_{C J}=\frac{\Gamma_{1} P_{0}}{\Gamma_{2}}\left(1-\sqrt{\left(1-\frac{\Gamma_{2}}{\Gamma_{1}}\right)\left(1+\frac{\Gamma_{2}}{\Gamma_{1}}+\frac{2 \Gamma_{2}}{\Gamma_{1}\left(\gamma_{1}+1\right)}\right)}\right) .
$$




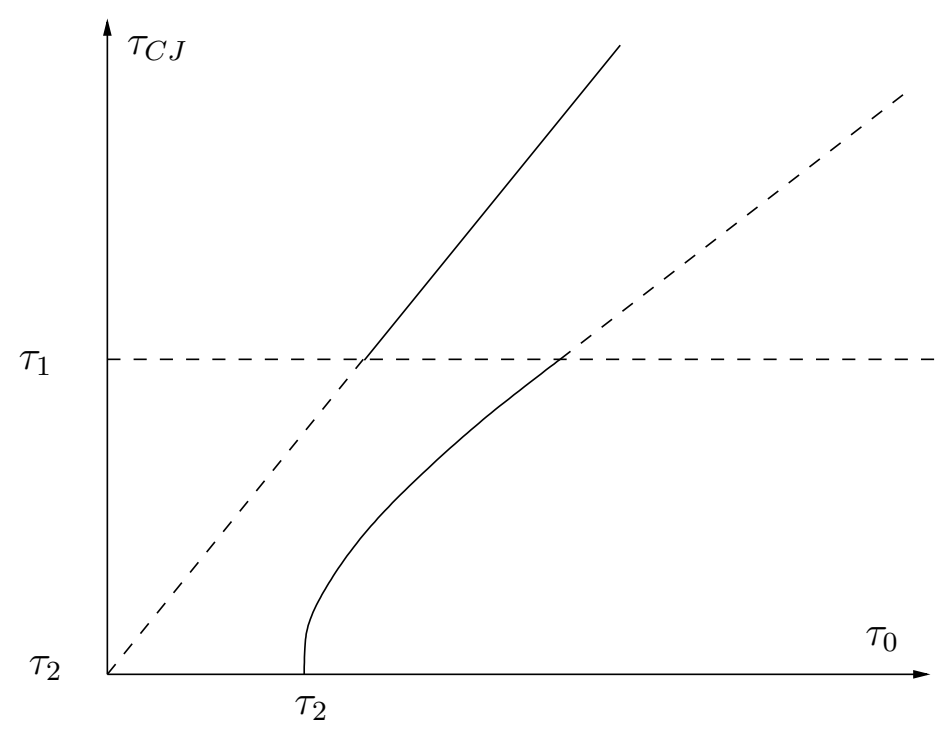

Figure 17: Qualitative behavior of the mixture and the vapor ChapmanJouguet points for the model with two perfect gas. The horizontal dashed line represents the vapor saturation curve. The increasing line (dashed line, then solid line) is the set of the Chapman-Jouguet points for the vapor equation of state. The other function (solid line, then dashed line) is the set of the mixture Chapman-Jouguet points. The solid lines of the curves correspond to the part in which they match with the equation of state used.

We remark that $P_{C J}$ is a linear function of $P_{0}$. If we use the equation of the Crussard curve, we get the following expression for $\tau_{C J}$

$$
\tau_{C J}=\tau_{0} \frac{\frac{\gamma_{2}+1}{\gamma_{2}-1} P_{0}+P_{C J}}{\frac{\gamma_{1}+1}{\gamma_{1}-1} P_{C J}+P_{0}} .
$$

As $P_{C J}$ is a linear function of $P_{0}$, we see that $\tau_{C J}$ is a linear function of $\tau_{0}$. The CJ-point of pure vapor can be chosen only when $\tau_{C J} \geq \tau_{1}$.

The two functions $\tau_{C J}$, for vapor and mixture equation of state are drawn on Figure 17, highlighting the fact that they cannot be linked continuously.

Example 2 : model with two stiffened gas

As already mentioned, the mixture equation of state cannot be computed when we deal with the two stiffened gas model. Therefore we can only show a numerical computation as an illustration. We chose the model of dodecane for which coefficients lie in Table 2. We begin on the point $P_{0}=900000 \mathrm{~Pa}$ with a specific volume of $\tau_{0}=0.0025 \mathrm{~kg} \cdot \mathrm{m}^{-3}$. We compute all the states $0^{\star}$ 


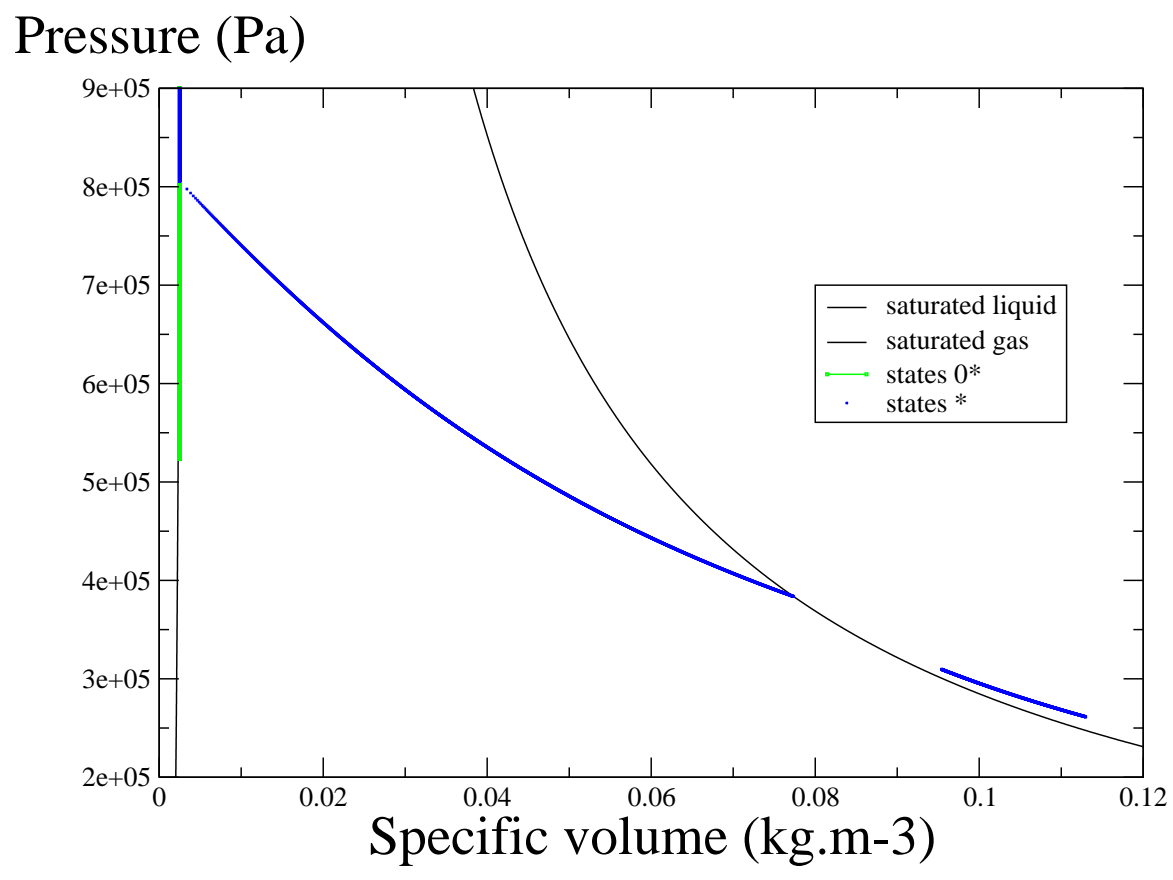

Figure 18: Set of all the Chapman-Jouguet points that can be reached from a given point. The isentrope is drawn in green, and is nearly vertical. In blue, the set of all the Chapman Jouguet points was drawn, which shows a jump between the mixture Chapman-Jouguet points and the pure vapor Chapman-Jouguet point.

that can be linked with that initial point via an isentrope. If the state $0^{\star}$ is overheated (i.e. lie in the saturation dome), then we compute the ChapmanJouguet point(s) corresponding to a mixture downstream state and/or to a pure vapor downstream state. Numerical results are on Figure 18.

\section{Conclusion}

In a first part we studied the model with two convex equation of state. In particular, we gave a necessary and sufficient condition for the convexity of the mixture equation of state resulting from an entropy optimization:

$$
\frac{\mathrm{d} P_{\text {sat }}}{\mathrm{d} T}>0
$$


Then we proposed to take into account metastable states in the solution of the Riemann problem. For that, we used the Chapman-Jouguet theory. We first proved that this theory can be applied. We emphasized the link between the overheat or overcooled of the metastable state and the retrograde and regular behavior of the fluid. In a particular case, when $\gamma / \Gamma$ does not depend on $\tau$, the condition of regular behavior of the fluid is necessary and sufficient to ensure that the energy of a metastable liquid is lower than the energy of a mixture at thermodynamic equilibrium with the same pressure and specific volume.

For the entropy growth condition, we proved that it is ensured provided $\gamma_{l}>\gamma_{m}$ and $\gamma_{l}>\gamma_{v}$.

The problem with the deflagration waves is that the Lax characteristic criterion is not ensured, so that the problem is under-determinated. The only thing that we can state with no more hypothesis is that the set of all the downstream states lies in an area limited on the top by the set of all the constant pressure deflagrations, which is continuous, and on below by the set of all the Chapman-Jouguet points, which was proved to be discontinuous thanks for a detailed study of the behavior of the Crussard curve near the saturation curve. As the set of all the Chapman-Jouguet points is discontinuous, the use of the Chapman-Jouguet closure as in [10] for solving the Riemann problem leads to a solution that does not depend continuously on its initial data in general. A first step to find a right kinetic closure would be for example to study travelling waves for relaxation model as given in [3]. As we know that liquid-vapor phase transition is governed by a competition between relaxation phenomena and thermal conduction, it would be more relevant (but much harder) to study travelling waves with relaxation model and thermal conductivity.

\section{Acknowledgements}

This work was partially supported by the CEA through the contract number 123-C-BEFI. The author would like to thank Siegfried Müller from Institut für Geometrie und Praktische Mathematik (Aachen, Germany) for profitable discussions on the Riemann problem with phase transition at thermodynamic equilibrium.

\section{References}

[1] Nabil Bedjaoui and Philippe G. LeFloch, Diffusive-dispersive traveling waves and kinetic relations. I. Nonconvex hyperbolic conservation laws, J. Differential Equations, 178 (2002), pp. 574-607.

[2] S. Benzoni-Gavage, Stability of subsonic planar phase boundaries in a van der Waals fluid, Arch. Ration. Mech. Anal., 150 (1999), pp. 23-55.

[3] Florian Caro, Modélisation et simulation numérique des transitions de phase liquide-vapeur, $\mathrm{PhD}$ thesis, École Polytechnique, CEA Saclay, November 2004. 
[4] R. Courant And K. O. Friedrichs, Supersonic Flow and Shock Waves, Interscience Publishers, Inc., New York, N. Y., 1948.

[5] Hai TAO FAn, A vanishing viscosity approach on the dynamics of phase transitions in van der Waals fluids, J. Differential Equations, 103 (1993), pp. 179-204.

[6] Edwige Godlewski and Pierre-Arnaud Raviart, Numerical approximation of hyperbolic systems of conservation laws, vol. 118 of Applied Mathematical Sciences, Springer-Verlag, New York, 1996.

[7] Philippe Helluy and Thomas Barberon, Finite volume simulation of cavitating flows, Computer \& Fluids, 34 (2005), pp. 832-858.

[8] Stephane JaOuen, Étude mathématique et numérique de la stabilité pour des modèles hydrodynamiques avec transition de phase, $\mathrm{PhD}$ thesis, Université Pierre et Marie Curie, CEA Bruyères-le-Châtel, November 2001.

[9] Olivier Le Métayer, Jacques Massoni, and Richard Saurel, Élaboration de lois d'état d'un liquide et de sa Vapeur pour les Modèles d'écoulements Diphasiques, Int. J. Thermal. Sci., 43 (2003), pp. 265-276.

[10] — Modelling evaporation fronts with reactive Riemann solvers, J. Comput. Phys., 205 (2005), pp. 567-610.

[11] TAi Ping LiU, The Riemann problem for general systems of conservation laws, J. Differential Equations, 18 (1975), pp. 218-234.

[12] Ralph Menikoff And Bradley J. Plohr, The Riemann problem for fluid flow of real materials, Rev. Modern Phys., 61 (1989), pp. 75-130.

[13] José Roberto Simões-Moreira and Joseph E. Shepherd, Evaporation waves in superheated dodecane, J. Fluid Mech., 382 (1999), pp. 63-86.

[14] M. Slemrod, Dynamic phase transitions in a van der Waals fluid, J. Differential Equations, 52 (1984), pp. 1-23.

[15] Philip A. Thomson, A fundamental derivative in gasdynamics, Phys. Fluids, 14 (1971), pp. 1843-1849.

[16] Philip A. Thomson, Garry C. Carofano, And Yoon-Gon Kim, Shock waves and phase changes in a large heat capacity fluid emerging from a tube, J. Fluid. Mech., 166 (1986), pp. 57-92.

[17] Philip A. Thomson, Humberto Chaves, G.E.A. Meier, Yoon-Gon KIm, AND H.D. SpECKMAN, Wave splitting in a fluid of large heat capacity, J. Fluid. Mech., 185 (1987), pp. 385-414.

[18] Philip A. Thomson And K. Lambrakis, Negative shock waves, J. Fluid. Mech., 60 (1973), pp. 187-208. 
[19] Alexander Voss, Exact Riemann solution for the Euler equations with Nonconvex and Nonsmooth Equation of state, $\mathrm{PhD}$ thesis, RheinischWestfälischen Teschnischen Hochschule Aachen, January 2005. available at http://www.it-voss.com/papers/thesis-voss-030205-128-final.pdf.

[20] Burton Wendroff, The Riemann problem for materials with nonconvex equations of state. I. Isentropic flow, J. Math. Anal. Appl., 38 (1972), pp. $454-466$.

[21] — The Riemann problem for materials with nonconvex equations of state. II. General flow, J. Math. Anal. Appl., 38 (1972), pp. 640-658.

[22] Ya. B. ZeL'Dovich And Yu. P. Raizer, Physics of shock waves and high temperature hydrodynamic phenomena, vol. II, Academic press, New York and London, 1967. 